\title{
Übergänge nach der Sekundarstufe I: ,Korrekturen von zugewiesenen Bildungswegen", aber Resultat langgehegter Bildungsaspirationen?
}

\author{
Nele Usslepp (i) · Franz Baeriswyl • Nicolas Hübner • \\ Benjamin Nagengast · Ulrich Trautwein
}

Online publiziert: 29. Mai 2019

(C) Der/die Autor(en) 2019

Zusammenfassung Im Deutschfreiburger Bildungssystem (Schweiz) basiert die Zuweisung nach der Grundschule (fast) ausschließlich auf einem standardisierten Leistungstest und einer Lehrerempfehlung. Hier getroffene Entscheidungen können an der nächsten Übertrittsschwelle korrigiert werden. Der vorliegende Beitrag untersuchte, inwieweit sowohl das Nachholen der Hochschulreife („Aufwärtskorrektur“) als auch das vorzeitige Verlassen des Weges zur Hochschulreife (,Abwärtskorrektur“) zwar „Korrekturen zugewiesener Bildungswege“ darstellen, aber gleichzeitig Resultat langgehegter Aspirationen sind. Zusätzlich wurde die Bedeutung von Aspirationen für die Zuweisung nach der Grundschule und den Schulerfolg (Noten in Klasse 9) untersucht. Die Datenbasis bildete die Vollerhebung zweier Übertrittsjahrgänge $(N=1219)$ an deutschsprachigen Schulen im Kanton Freiburg. Die Ergebnisse der (logistischen) Regressionsmodelle zeigten, dass die Aspirationen von Eltern und Kindern (gemessen in der Grundschule) die Korrekturen am Ende der Sekundarstufe I und den Schulerfolg in der 9. Klasse vorhersagten. Der Übergang nach der Sekundarstufe I stellt zwar die „Korrektur von zugewiesenen Bildungswegen“ dar, aber ist gleichzeitig das Resultat langgehegter Aspirationen.

\footnotetext{
N. Usslepp $(\varangle) \cdot$ Dr. N. Hübner · Prof. Dr. B. Nagengast · Prof. Dr. U. Trautwein Hector-Institut für Empirische Bildungsforschung, Universität Tübingen, Europastr. 6, 72072 Tübingen, Deutschland E-Mail: nele.usslepp@uni-tuebingen.de

Dr. N. Hübner

E-Mail: nicolas.huebner@uni-tuebingen.de

Prof. Dr. B. Nagengast

E-Mail: benjamin.nagengast@uni-tuebingen.de

Prof. Dr. U. Trautwein

E-Mail: ulrich.trautwein@uni-tuebingen.de

Prof. Dr. F. Baeriswyl

Universität Freiburg, Freiburg, Schweiz

E-Mail: franz.baeriswyl@unifr.ch
} 
Schlüsselwörter Aspirationen · Bildungsentscheidung · Deutschfreiburger (Schweiz) Bildungssystem · Korrektur · Übergang

\title{
Transitions after lower secondary education: "Corrections of allocated educational paths", but the result of long-standing aspirations?
}

\begin{abstract}
In the Deutschfreiburg (Swiss) educational system, the paths that students follow after primary school are (almost) exclusively based on standardized performance tests and teacher recommendations. However, decisions made at this point can be corrected at the next transition. Thus, in the present study, we analyzed whether changing from intermediate track to higher education ("upward correction") and dropping out of higher education ("downward correction") are "corrections of allocated educational paths" but reflect long-standing aspirations. In addition, we investigated the influence of aspirations on the path allocated after primary school as well as on educational success (grades earned in the 9th grade). Data from all German-speaking schools in the Canton of Freiburg $(N=1219$ students in two cohorts) were used. The results of (logistic) regression models showed that parents' and children's aspirations (measured in primary school) predicted both corrections and grades. Thus, the transition after lower secondary school represents "corrections of allocated educational paths" but are also the result of long-standing aspirations.
\end{abstract}

Keywords Aspirations - Educational Decision - Educational System of Deutschfreiburg (Swiss) · Correction · Transition

\section{Einleitung}

Seit den 1970er Jahren gab es in Deutschland und der Schweiz viele bildungspolitische Anstrengungen, die zur Öffnung und damit zur erhöhten Durchlässigkeit im Bildungssystem führten. Durch diesen wichtigen Aspekt der Modernisierung des Schulsystems sollte die Tragweite der frühen Leistungsdifferenzierung begrenzt werden und Schüler/-innen folglich die Möglichkeit erhalten, eingeschlagene oder zugewiesene Bildungswege korrigieren zu können (Deutscher Bildungsrat 1970; EDK 1970). Auf- und Abwärtskorrekturen sollen dann stattfinden, wenn die individuellen Leistungen nicht den institutionellen Anforderungen entsprechen (Oesch 2017). Darüber hinaus erhofft man sich durch diese Korrekturmöglichkeiten eine Reduktion des Zusammenhangs von sozialer Herkunft und der Bildungsbeteiligung, der vielseitig nachgewiesen wurde (z.B. Harazd und Ophuysen 2008; Maaz und Nagy 2009; Dumont et al. 2014).

In der bildungswissenschaftlichen Literatur haben sich mittlerweile verschiedene theoretische Modelle zur Vorhersage von Bildungsentscheidungen etabliert (z. B. Boudon 1974; Erikson und Jonsson 1996; Breen und Goldthorpe 1997). Besonders relevant für die vorliegende Studie ist das Modell der Wisconsin-Schule (z. B. Sewell et al. 1970), da es eine Vielzahl an wichtigen Faktoren inkludiert und Bildungs- bzw. Karriereverläufe auf eine längere Sicht betrachtet. Von zentraler Bedeutung sind in diesem Modell Bildungsaspirationen, die nach der Wisconsin-Schule als frühzeitig 
festgelegt und zeitstabil gelten (Becker 2010). Der Einfluss von Aspirationen auf unterschiedliche Indikatoren von Bildungsverläufen wurde bereits des Öfteren aufgezeigt (z. B. Hauser 2005). Allerdings fehlen fundierte Erkenntnisse darüber, welche Rolle langehegte Aspirationen bei der Nutzung der Öffnungsoptionen übernehmen. In Abhängigkeit von den jeweiligen Übergangsregelungen nach der Grundschule sind unterschiedliche Einflüsse von Aspirationen denkbar: In Bildungssystemen mit „freier“ Übertrittswahl können Aspirationen direkt realisiert werden. Dagegen könnte es in Systemen mit einer Zuweisung nach der Grundschule dazu kommen, dass Eltern und Schüler/-innen nicht direkt ihre Aspirationen realisieren können, diese aufrechterhalten - im Sinne des Wisconsin-Modells - und die Öffnungsoption nutzen, um ihren frühzeitig festgelegten Bildungswunsch umzusetzen.

Ziel der vorliegenden Studie ist es, die Bedeutung langgehegter Aspirationen in einem Bildungssystem zu prüfen, in dem die vertikale Öffnung (Übergang nach der Sekundarstufe I) die erste ,freie“ Bildungsentscheidung von Eltern und Schüler/ -innen darstellt. Dazu werden theoretische Annahmen des Wisconsin-Modells auf die Besonderheiten des Deutschfreiburger Schulsystems - aus dem die Daten der vorliegenden Studie stammen - übertragen. Anhand von Längsschnittdaten wird zunächst untersucht, ob bereits der Übergang nach der Grundschule (trotz eines streng geregelten Zuweisungsverfahrens; Forschungsfrage 1) und der Schulerfolg in der Sekundarstufe I (Forschungsfrage 2) durch die Aspirationen von Eltern und Kindern beeinflusst werden. Anschließend beschäftigt sich die Hauptfragestellung damit, ob mit bereits am Ende der Grundschule gefassten Aspirationen von Eltern und Kindern sowohl das Nachholen der Hochschulreife durch einstige Schüler/-innen der Sekundarabteilung rsp. Realschule als auch das vorzeitige Verlassen des Weges zur Hochschulreife (einstige Schüler/-innen von der progymnasialen Abteilung rsp. Gymnasium) vorhergesagt werden kann (Forschungsfrage 3).

\section{Theoretischer Hintergrund}

\subsection{Institutioneller Hintergrund und Ungleichheiten bei der Bildungspartizipation}

Nach dem meritokratischen Prinzip sollte die Leistungsfähigkeit die entscheidende Determinante für den Bildungsweg nach der Grundschule sein (Becker und Hadjar 2017). Für die Schweiz und Deutschland ist jedoch hinlänglich bekannt, dass auch weitere Faktoren die Zuweisungsentscheidungen zu den Schultypen der Sekundarstufe I beeinflussen: der sozioökonomische Hintergrund, der Migrations- und der Sprachhintergrund des Kindes (z. B. Deutsches PISA-Konsortium 2001; Maaz et al. 2011; Becker und Zangger 2013). Zudem ist die Prognose der Leistungsfähigkeit nach dem Übertritt in die Sekundarstufe I aufgrund ungenauer Leistungsdiagnostik sowie diskontinuierlicher Entwicklungsverläufe mit einem relativ hohen Fehler behaftet (z. B. Baumert et al. 2003b, 2009), weshalb sich Übertrittsentscheidungen als „falsch“ erweisen können.

Um die Tragweite eines eingeschlagenen bzw. zugewiesenen Bildungsweges zu begrenzen, sollen Öffnungsoptionen im Bildungssystem ermöglichen, früher getrof- 
fene Übertrittsentscheidungen korrigieren zu können (Deutscher Bildungsrat 1970; siehe auch EDK 1970; BMBF 2010). Die Durchlässigkeit im Schweizer Bildungssystem ist seit 2006 sogar in der Bundesverfassung verankert (Bundesverfassung 1999 [Stand: 2018]). Für die Schweiz wurde jedoch aufgezeigt, dass sich Korrekturen innerhalb der Sekundarstufe I als äußerst schwierig erweisen - lediglich 4-6\% nutzen die sogenannte horizontale Öffnung (z. B. Sacchi et al. 2011). Dagegen hat sich der Übergang nach dem Durchlaufen der Sekundarstufe I als eine Gelenkstelle erwiesen, an der viele Korrekturen stattfinden (für die Schweiz: z. B. Sacchi et al. 2011; Oesch 2017; für Deutschland: Henz und Maas 1995; Buchholz et al. 2016).

Mit dem Ziel den Übertritt in die Sekundarstufe I möglichst nach dem meritokratischen Prinzip zu gestalten, können Eltern und Kinder im Kanton Freiburg (Schweiz), aus dem die in diesem Artikel analysierten Daten stammen, keine „freie“ Bildungsentscheidung treffen. Schüler/-innen werden nach der Grundschule (nach der 6. Klasse) vielmehr (fast) ausschließlich auf Grundlage institutioneller Vorgaben (eines standardisierten Leistungstests und der Lehrerempfehlung) in die weiterführende Schule zugewiesen (Baeriswyl et al. 2006). Allerdings können auch bei diesen Zuweisungselementen gewisse Probleme mit dem Meritokratieprinzip auftreten (z.B. Referenzgruppeneffekte bei Lehrerempfehlungen; Trautwein und Baeriswyl 2007). Hofstetter (2017) stellte darüber hinaus in einer qualitativen Studie fest, ,... dass die Schule als Organisation Eigeninteressen verfolgt und als eigensinniger Akteur Schülerströme lenkt“ (S. 292). In Deutschfreiburg können Schüler/ -innen aus der progymnasialen Abteilung (entspricht in Deutschland dem Gymnasium) nach der Sekundarstufe I (nach der 9. Klasse) mit einem Notendurchschnitt von „genügend“ den Übertritt in das vierjährige Obergymnasium vornehmen und ihre Matura (Hochschulreife) erreichen. Schüler/-innen der Sekundarabteilung (im Folgenden „,Realschule“ genannt) mit einem Notendurchschnitt ,gut“ in den Hauptfächern können ebenfalls prüfungsfrei ins Obergymnasium übertreten. Die Übrigen haben die Möglichkeit, sich nach dem neunten Schuljahr einer Aufnahmeprüfung für den Eintritt in das Obergymnasium zu stellen. Des Weiteren besteht für alle Schüler/-innen die Möglichkeit, die Berufsmatura (fachgebundene Hochschulreife) zu erlangen. Schüler/-innen aus der Realabteilung (entspricht der Hauptschule in Deutschland) haben theoretisch dieselben Möglichkeiten, diese werden allerdings sehr selten genutzt - deshalb findet diese Gruppe im vorliegenden Beitrag keine weitere Berücksichtigung. Trautwein et al. (2008) konnten für die Öffnungsoption im Anschluss an die Sekundarstufe I in Deutschfreiburg (dem deutschsprachigen Teil des Kantons Freiburg) zeigen, dass $22 \%$ der Schüler/-innen der Realschule in den gymnasialen Weg wechselten. Diese Nutzung war jedoch auch durch die soziale Herkunft geprägt.

\subsection{Die Rolle von Aspirationen bei Bildungsverläufen}

Neben den schulischen Leistungen eines Kindes sind Bildungsaspirationen der wichtigste Prädiktor für Bildungsentscheidungen und -verläufe (z. B. Becker 2000). Das Konzept der Aspirationen und dessen theoretische Grundlage ist in den 1960ern und 70er Jahren durch die Wisconsin-Schule bekannt geworden (z. B. Sewell et al. 1970). Nach der Wisconsin-Schule sind Aspirationen ein , cognitive orientational aspect of 
goal-directed behavior" (Haller 1968, S. 484). In der aktuellen wissenschaftlichen Literatur wird teilweise eine analytische Trennung zwischen realistischen und idealistischen Aspirationen vorgenommen, d.h. zwischen erwarteten Bildungsabschlüssen und Bildungswünschen (Haller 1968; siehe auch Becker 2010). In der vorliegenden Studie wird der Einfluss der idealistischen Aspirationen (Bildungswünsche) fokussiert. Nach Zimmermann (2018) sind die Bildungswünsche bedeutender für Bildungsverläufe, da sie eine motivationale Komponente enthalten und neben Entscheidungen auch die realistischen Aspirationen beeinflussen (Zimmermann 2018, S. 343). Üblicherweise werden idealistische Aspirationen erfasst durch Items wie „Welchen Schulabschluss wünschen Sie sich für Ihr Kind?“”.

Mit Blick auf Bildungsentscheidungen und -verläufe werden unterschiedliche theoretische Ansätze diskutiert. In diesem Zusammenhang sind vor allem zwei Erklärungsansätze anzuführen (siehe Kleine et al. 2009), die unterschiedliche Sichtweisen auf Aspirationen haben: die Rational Choice Theorie und das WisconsinModell des Statuserwerbs.

Die Rational Choice Theorie - deren prominentester Vertreter Boudon (1974) ist geht davon aus, dass alle Personen grundsätzlich die gleichen Präferenzen bezüglich Bildungsabschlüsse haben. Unterschiede in Bildungsentscheidungen und -aspirationen (auch bezeichnet als „,vorweggenommene Bildungsentscheidungen“; Kurz und Paulus 2008, S. 5490) lassen sich somit durch bewusste unterschiedliche KostenNutzen-Kalkulationen erklären. So wägen beispielsweise Eltern ab, welche Kosten mit der Entscheidung für einen bestimmten Bildungsgang einhergehen und welcher mögliche Nutzen aus dieser Entscheidung resultieren könnte. Die Bewertung einer Entscheidungssituation unterliegt sowohl zeitkonstanten als auch zeitvariablen Einflussfaktoren. Nach diesem Ansatz wird zum Beispiel die schulische Leistung des Kindes, die als zeitveränderlich gilt, sowie der Bildungshintergrund (gilt als zeitkonstant) berücksichtigt, um eine Bildungsentscheidung zu treffen (siehe Kleine et al. 2009). Nach der Rational Choice Theorie werden idealistische und realistische Aspirationen daher als eher zeitvariabel betrachtet, die je nach Entscheidungssituation unterschiedlich ausfallen können (Kurz und Paulus 2008).

Der zweite Erklärungsansatz entstammt der Wisconsin-Schule (z. B. Sewell et al. 1970). Das Wisconsin-Modell des Statuserwerbs geht - im Gegensatz zur Rational Choice Theorie (z. B. Boudon 1974) - davon aus, dass Personen grundsätzlich unterschiedliche Aspirationen haben, die das Resultat von Normen, Werte und Leistungsziele des sozialen Umfelds (,,significant others“) sind. Aspirationen werden in diesem Modell zu einem großen Teil auf unbewusste Bezugsgruppeneinflüsse zurückgeführt. Häufig werden zur Berücksichtigung des sozialen Umfeldes - neben den Eltern - auch die Aspirationen von Freunden oder Lehrkräften einbezogen (z. B. Zimmermann 2018). Mit Blick auf die vorliegende Studie könnten beispielsweise die elterlichen Aspirationen einen Einfluss auf den weiteren schulischen Erfolg oder den Bildungsverlauf haben (Haller und Portes 1973). Des Weiteren werden Aspirationen im Wisconsin-Modell als eher zeitstabil angesehen (z. B. Sewell et al. 1970; Gambetta 1996). Das bedeutet, dass Entscheidungssituationen selbst nur eine untergeordnete Bedeutung zukommt, da der Bildungsweg des Kindes schon frühzeitig geplant und festgelegt ist (Kleine et al. 2009; S. 106). 
Paulus und Blossfeld (2007) untersuchten diese beiden Erklärungsansätze mit Blick auf die Entscheidungssituation nach der Grundschule. Sie zeigten für die realistischen Bildungsaspirationen der Eltern, dass sowohl die angenommenen Einflüsse der Rational Choice Theorie als auch des Wisconsin-Modell aufgetreten sind. Dementsprechend hatten die idealistischen Aspirationen einen signifikanten Einfluss auf die Bildungsentscheidung am Ende der Grundschule - unter Kontrolle des Bildungshintergrunds und der schulischen Leistung des Kindes. Darüber hinaus liegen Studien vor, die sich mit der Stabilität von elterlichen Aspirationen, ausgehend von beiden Erklärungsansätzen, beschäftigen. So stellten beispielsweise Kleine et al. (2009) fest, dass ein knappes Drittel der Eltern ihre realistischen Bildungsaspirationen vom dritten bis zum vierten Schuljahr des Kindes aufrechterhielten (siehe auch Ditton und Krüsken 2010). Die vorliegende Untersuchung fokussiert weder die Formation von Aspirationen noch eine einzelne Entscheidungssituation. Vielmehr interessieren wir uns dafür, ob frühzeitig manifeste Aspirationen einen langfristigen Einfluss auf Bildungsverläufe haben.

Neben der Erklärung von Aspirationen und schulischen Leistungen, betrachtet das Wisconsin-Modell auch die Einflüsse eben dieser auf Bildungsverläufe (Becker 2010). Im Ausgangsmodell der Wisconsin-Schule wird nicht nach den unterschiedlichen Aspirationsarten differenziert und mögliche Einflüsse des Bildungssystems werden ebenfalls nicht berücksichtigt. Becker (2010) geht jedoch davon aus, dass sowohl realistische als auch idealistische Aspirationen einen direkten Einfluss auf Bildungsverläufe haben und sich die Bildungswünsche nicht durch institutionelle Bedingungen ändern (S. 16). Mit Blick auf die vorliegende Studie kann somit vermutet werden, dass sich Eltern und ihre Kinder nicht durch eine Zuweisung nach der Grundschule von ihrem frühzeitig aspirierten Bildungsweg abbringen lassen. Folglich sollten Eltern und Schüler/-innen versuchen ihre idealistischen Aspirationen, die mit einer Zuweisung nach der Grundschule nicht erfüllt wurden, bei der nächsten Möglichkeit (z. B. vertikale Öffnung) zu verwirklichen (vgl. Becker 2010).

Die Annahmen der Wisconsin-Schule hinsichtlich der Formationen von Aspirationen sind empirisch gut bestätigt (z. B. Hauser 2005; für einen Überblick: Sewell et al. 2003). So sind die Einflüsse des sozialen Umfeldes (z. B. Zimmermann 2018) und des sozialen Hintergrundes (z. B. Ditton et al. 2005) auf Aspirationen gut erforscht. Beispielsweise zeigen Eltern mit einem höheren sozialen Hintergrund höhere Aspirationen (z. B. Ditton et al. 2005). Diese Differenzen bleiben auch nach Kontrolle der schulischen Leistung des Kindes bestehen. Mit Blick auf den langfristigen Einfluss von Aspirationen auf Bildungsverläufe finden sich jedoch bisher kaum Studien mit Stichproben aus Deutschland oder der Schweiz. Dies scheint vor allem den fehlenden längsschnittlich angelegten Studien geschuldet zu sein (siehe Ditton 2013). Erste Hinweise für den Einfluss von langfristigen elterlichen Erwartungen liefert eine Untersuchung von Neuenschwander und Malti (2009), die eine Stichprobe aus dem Kanton Bern (Schweiz) verwendet. Sie konnten aufzeigen, dass die Bildungserwartungen der Eltern in der sechsten Klasse (Ende der Grundschule) einen signifikanten Einfluss für den weiteren Bildungsweg (Gymnasium versus Berufsausbildung) im Anschluss an die Sekundarstufe I hatten. Des Weiteren ist eine Studie von Wohlkinger und Ditton (2012) hervorzuheben, die analysierte, ob Schüleraspirationen eine Vorhersagekraft für den Übergang nach der Grundschule haben - unter Kontrolle der 
Elternaspirationen und der Lehrerempfehlung. Die Ergebnisse zeigten zum einen, dass sich Eltern- und Schüleraspirationen trotz starken Zusammenhangs voneinander abgrenzen lassen und zum zweiten, dass die Eltern- und Schüleraspirationen mit der Schulanmeldung in der Sekundarstufe I zusammenhingen. Erstaunlich ist, dass kindliche Aspirationen ansonsten eher selten in der aktuellen Übergangsforschung inkludiert werden, während die Wirkung von elterlichen Aspirationen des Öfteren im Fokus von Untersuchungen ist (z. B. Becker 2000). Exemplarisch zu nennen ist hier die Studie von Stamm (2005). Sie zeigte für eine Stichprobe aus der Schweiz, dass sich elterliche Aspirationen auf die Schulleistung der Kinder auswirkten.

\section{Die Fragestellungen der vorliegenden Studie}

Längsschnittliche Untersuchungen, die sowohl den Grundschulübergang als auch die mögliche Nutzung vertikaler Öffnungsoptionen nach der Sekundarstufe I berücksichtigen, sind rar. Die wenigen existierenden Forschungsarbeiten verwenden retrospektiv erfasste Daten (z.B. Schneider 2008) - weshalb kaum Möglichkeiten bestehen, Wirkungen, Ursachen oder Bedingungen von Bildungsverläufen aufzuklären (vgl. Ditton 2013). Unseres Wissens nach haben lediglich Neuenschwander und Malti (2009) sowohl den Übertritt nach der Grundschule als auch nach der Sekundarstufe I berücksichtigt. Die vorliegende Studie prüft anhand von Längsschnittdaten den langfristigen Einfluss von idealistischen Aspirationen auf Bildungsverläufe. Dabei dient das Wisconsin-Modell als theoretische Grundlage für angenommene Wirkmechanismen und wird auf die Besonderheiten des Deutschfreiburger Systems übertragen. Konkret werden drei Forschungsfragen und deren Hypothesen analysiert:

Forschungsfrage 1 Zunächst prüfen wir, ob die idealistischen Aspirationen von Eltern und Kindern die Zuweisung nach der Grundschule in Deutschfreiburg beeinflussen. Insbesondere in Schulsystemen mit bindender Zuweisung (wie in Deutschfreiburg) sollten Eltern- und Schüleraspirationen aufgrund der institutionellen Reglementierungen keinen Zusammenhang mit dem Übergang nach der Grundschule aufweisen, wenn die Zuweisungselemente (Lehrerempfehlung und Vergleichsprüfung) kontrolliert werden (Hypothese 1).

Forschungsfrage 2 Des Weiteren untersuchen wir, ob sich die idealistischen Aspirationen der Eltern und Kinder auf die Leistungen am Ende der Sekundarstufe I auswirken. Wir vermuten, dass der Schulerfolg in der Sekundarstufe I sowohl durch die Eltern- als auch die Schüleraspirationen beeinflusst wird (Hypothese 2).

Forschungsfrage 3 Schließlich überprüfen wir, ob die vertikale Öffnung des Bildungssystems in Deutschfreiburg - im Anschluss an die Sekundarstufe I - dazu genutzt wird, frühe idealistische Bildungsaspirationen umzusetzen. Eltern und ihre Kinder, die nicht die erhoffte Zuweisung erhalten haben, könnten seit der Grundschule den Plan verfolgen, den Übergang nach der Sekundarstufe I zu nutzen, um auf eine höhere Schulform zu wechseln („Aufwärtskorrektur“). Demnach erwarten 
wir, dass die Aufwärtskorrektur mit bereits am Ende der Grundschule gefassten höheren Bildungswünschen der Schüler/-innen und ihrer Eltern einhergeht (Hуроthese 3a). Ähnliches könnte auch für Schüler/-innen und Eltern gelten, die zwar eine Zuweisung zum Gymnasium erhalten haben, diese aber nur nutzen wollten, um eine ,bessere“ mittlere Reife zu erlangen (vgl. Winkler 2016). Schüler/-innen aus dem Gymnasium entscheiden am Ende der Sekundarstufe I, ob sie vorzeitig den Weg zur Hochschulreife verlassen („Abwärtskorrektur“). Wir nehmen an, dass die Abwärtskorrektur mit einer bereits am Ende der Grundschule gefassten tieferen idealistischen Bildungsaspiration einhergeht (Hypothese 3b).

\section{Methode}

\subsection{Stichprobe}

Die Datengrundlage der Analysen bildete die Vollerhebung zweier Übertrittsjahrgänge aus Deutschfreiburg. Die erste Befragung wurde im Jahr 2009 (,Kohorte 2009“) bzw. 2010 („Kohorte 2010“) durchgeführt. Die Schüler/-innen wurden vor der Vergleichsprüfung schriftlich befragt. Die Vergleichsprüfung ist ein standardisierter Leistungstest, der für alle Schüler/-innen obligatorisch war und nach dem ersten Semester des 6. Schuljahres (= letztes Schuljahr der Grundschule), im Monat März, stattfand. Die Eltern wurden nach Bekanntgabe des Zuweisungsentscheides schriftlich befragt. Diese erste Erhebung enthält Daten von insgesamt 1684 Schüler/-innen, wovon 51\% weiblich waren, und von 1406 Eltern. Nach Ausschluss der Hauptschüler/-innen, denen nach der Sekundarstufe I praktisch nie ein direkter Übertritt auf das Gymnasium gelingt (siehe oben), umfasste die hier berücksichtige Stichprobe $N=1219$ Personen ( $N=469$ Gymnasiasten/-innen; $N=750$ Realschüler/ -innen), davon 50,7\% Mädchen.

Am Ende des neunten Schuljahres (= Ende der Sekundarstufe I) fand eine zweite schriftliche Befragung der Schüler/-innen statt. Zu demselben Zeitpunkt wurden alle Zeugnisnoten aller Semester der dreijährigen Sekundarstufe I erfasst. Die berücksichtigte Stichprobe umfasste für diesen zweiten Messzeitpunkt insgesamt $N=1051$ Schüler/-innen ( $N=626$ Realschüler/-innen und $N=425$ Gymnasiasten).

Im Rahmen eines weiteren Forschungsprojektes wurde die Kohorte 2010 (nicht aber die Kohorte 2009) drei Monate nach vollzogenem Übergang in die Sekundarstufe II elektronisch nachbefragt (3. Messzeitpunkt). Diese Beschränkung erklärt die - trotz hoher Ausschöpfungsrate der Kohorte 2010 von $81 \%$ Prozent - relativ kleine Stichprobe von $N=502$ ( $N=315$ Realschüler/-innen; $N=187$ Gymnasiasten) zum dritten Messzeitpunkt. 
Tab. 1 Deskriptive Befunde für alle Variablen

\begin{tabular}{|c|c|c|c|c|c|}
\hline Variable & $N$ & M & SD & Min & $\operatorname{Max}$ \\
\hline \multicolumn{6}{|l|}{ Lehrervariable } \\
\hline Empfehlung & 1219 & 3,75 & 0,99 & 1 & 5 \\
\hline \multicolumn{6}{|l|}{ Schülervariablen } \\
\hline Prüfungsleistung Kl. 6 & 1217 & 70,04 & 9,47 & 40 & 93 \\
\hline Aspiration K1. 6 & 953 & 0,61 & 0,48 & 0 & 1 \\
\hline Notenschnitt Kl. 9 & 1090 & 48,94 & 3,73 & 37,50 & 59,00 \\
\hline \multicolumn{6}{|l|}{ Elternvariablen } \\
\hline Empfehlung & 1219 & 3,89 & 0,98 & 1 & 5 \\
\hline HISEI & 1204 & 54,27 & 15,98 & 18 & 89 \\
\hline Bildungsabschluss & 1055 & 0,61 & 0,49 & 0 & 1 \\
\hline Sprachgebrauch & 1093 & 0,90 & 0,30 & 0 & 1 \\
\hline Aspiration K1. 6 & 1051 & 0,41 & 0,49 & 0 & 1 \\
\hline Zuweisung (Gymnasium) & 1219 & 0,39 & 0,49 & 0 & 1 \\
\hline \multicolumn{6}{|l|}{ Korrekturen } \\
\hline $\begin{array}{l}\text { Beabsichtigte Aufwärtskorrek- } \\
\text { tur }\end{array}$ & 626 & 0,43 & 0,49 & 0 & 1 \\
\hline $\begin{array}{l}\text { Tatsächliche Aufwärtskorrek- } \\
\text { tur }\end{array}$ & 315 & 0,29 & 0,45 & 0 & 1 \\
\hline $\begin{array}{l}\text { Beabsichtigte Abwärtskorrek- } \\
\text { tur }\end{array}$ & 425 & 0,23 & 0,42 & 0 & 1 \\
\hline Tatsächliche Abwärtskorrektur & 187 & 0,40 & 0,49 & 0 & 1 \\
\hline
\end{tabular}

$N$ Anzahl an Teilnehmer/-innen, die Angaben auf der Variable gemacht haben, $M$ Mittelwert, $S D$ Standardabweichung, Min Minimum, Max Maximum, HISEI Höchster sozioökonomischer Status

\subsection{Instrumente}

Die verwendeten Variablen und ihre deskriptiven Befunde finden sich in Tab. 1.

\subsubsection{Kriteriumsvariablen}

Aufwärtskorrektur Die Realschüler/-innen haben im Anschluss an die Sekundarstufe I die Möglichkeit den Weg zur Hochschulreife einzuschlagen. Um die Breite dieser Übergangsentscheidung abzubilden und die Ergebnisse auf ihre Stabilität zu prüfen, wurde sowohl die „,beabsichtigte“ (für beide Kohorten vorliegend) als auch die „tatsächliche“ (für Kohorte 2010 vorliegend) Entscheidung zur Aufwärtskorrektur in den Analysen berücksichtigt. Zur Operationalisierung wurde die International Standard Classification of Education (ISCED) der UNESCO (2012; EDK 2015a) verwendet. Wie diese Klassifikation vorgibt, wurde zwischen berufsbildenden (ISCED 35) und allgemeinbildenden Anschlusslösungen (ISCED34) differenziert (siehe für eine Übersicht: EDK 2015b; Oesch 2017): Alle Schüler/-innen der Realschule, welche eine Anschlusslösung zur Erlangung einer Hochschulreife (allgemeinbildende Anschlusslösung: gymnasiale Matura; Berufsmatura; Fachmittelschul-Matura) wählten bzw. beabsichtigten, erhielten eine Kodierung „1“ (,,Korrektur"). Die Kodierung „0“ entspricht „,keine Korrektur“. Diese erhielten alle, die 
eine berufsbildende Anschlusslösung (z. B. Berufslehre, Anlehre, Praktikum) beabsichtigten bzw. wählten und somit nicht direkt den Weg zu einer Hochschulreife einschlugen.

Abwärtskorrektur Die Gymnasiasten können im Anschluss an die Sekundarstufe I entscheiden, ob sie den Weg zur Hochschulreife vorzeitigt verlassen wollen. Hier wurde ebenfalls sowohl die beabsichtigte als auch die tatsächliche Entscheidung zur Abwärtskorrektur berücksichtigt. Zur Operationalisierung wurde auch hier der ISCED der UNESCO (2012; EDK 2015a) herangezogen. Somit wurde hier ebenfalls die Differenzierung in allgemeine (ISCED34) und berufsbildende Anschlusslösung (ISCED35) vorgenommen und folglich die Abwärtskorrektur gebildet (siehe für eine Übersicht: EDK 2015b; Oesch 2017): Kodierung „1“ (= „Korrektur“) erhielten alle Schüler/-innen des Gymnasiums, die direkt nach der Sekundarstufe I den Weg zur Hochschulreife verließen bzw. verlassen wollten (z. B. Schüler/-innen, die eine Berufslehre begannen). Eine „,0“-Kodierung entspricht ,keiner Korrektur“ und diese erhielten alle, die weiter den Weg zu einer Hochschulreife direkt gehen wollten bzw. gingen (gymnasiale Matura, Berufs- oder Fachmittelschul-Matura).

Zuweisung Die zugewiesene Schulform im Anschluss an die Grundschule wurde als dichotome Variable in den Analysen berücksichtigt (1=,Gymnasium“; $0=$, ,andere“). Die Kategorie ,,andere“ umfasste die Hauptschule und die Realschule.

Notenschnitt Klasse 9 Der Schulerfolg in der Sekundarstufe I wurde durch den Notenschnitt der 9. Klasse operationalisiert. Die Noten wurden den Schulakten entnommen. Der Notenschnitt am Ende der Sekundarstufe I wurde dann wie folgt gebildet: Deutsch, Mathematik, und Französisch zählen jeweils doppelt; Englisch, Geographie, Geschichte und Naturlehre zählen einfach. Noten im Schulsystem in Deutschfreiburg werden im Wertebereich von 6 (sehr gut), 5 (gut), 4 (genügend), 3 (ungenügend), und 2 (schlecht) vergeben. Halbe Noten sind möglich. Höhere Zahlenwerte entsprechen also höheren Leistungen.

\subsubsection{Prädiktoren - Elternvariablen}

Elterliche Aspiration Klasse 6 Zentral für unser Forschungsvorhaben ist die elterliche Aspiration. Am Ende der Grundschule wurden die Eltern gebeten anzugeben: „Welchen Bildungsabschluss wünschen Sie sich für Ihr Kind?“. Die Angaben sind dichotomisiert in den Analysen berücksichtigt worden $(0=$, unter Universitäts-, Fachhochschul-oder PH-abschluss “; 1 = „,mindestens Universitäts-, Fachhochschuloder PH-abschluss“). Beispielsweise wurde auf der elterlichen Aspiration eine „1“ kodiert, wenn sich die Eltern für ihr Kind das „Doktorat“ wünschten und eine „0“ wurde zugewiesen, wenn die Eltern den Abschluss der Sekundarstufe I für ihr Kind aspirierten.

Elternempfehlung Auf einer 5-stufigen Skala gaben die Eltern eine Zuweisungsempfehlung nach der Grundschule ab - die Empfehlung der Lehrkräfte war ihnen 
vorher bekannt ( 5 = „, Gymnasium empfohlen “; 4 = ,Gymnasium bedingt empfohlen “; $3=$, Realschule empfohlen "; 2 = , Realschule bedingt empfohlen “; 1 = „Hauptschule empfohlen"). Die Empfehlung der Eltern wird jedoch nur bei der Zuweisungsentscheidung berücksichtigt, wenn die Lehrerempfehlung und die Vergleichsprüfung nicht zum selben Resultat führten. Diese Angabe wurde zum ersten Messzeitpunkt erfasst.

Sozioökonomischer Status der Eltern Sofern Angaben zum Beruf von den befragten Eltern vorlagen, wurden diese in den Internationalen Sozioökonomischen Index (ISEI; Ganzeboom und Treiman 1996) transformiert - fehlten entsprechende Angaben, wurden die Angaben der Schüler/-innen herangezogen. Der jeweils höhere ISEI-Wert (HISEI) der beiden Elternteile wurde als Wert für den sozioökonomischen Hintergrund des jeweiligen Schülers/der jeweiligen Schülerin verwendet. Der HISEI kann Werte zwischen 16 (z.B. Reinigungskraft) und 90 (z.B. Richter) annehmen.

Sprachgebrauch Der Sprachgebrauch in der Familie wurde im Schülerfragebogen zum ersten Messzeitpunkt erfasst. In den Analysen wurde eine dichotomisierte Variable berücksichtigt $(1=$, Deutsch“; $0=$, andere Sprache“ $)$.

Bildungshintergrund Im Hinblick auf den Bildungshintergrund wurde zwischen Familien, bei denen mindestens ein Elternteil die Hochschulzugangsberechtigung erworben hat $(1=$,hoher Bildungshintergrund") und den übrigen Familien $(0=$, ,niedriger Bildungshintergrund" $)$ unterschieden. Die Angaben wurden dem Elternfragebogen entnommen.

\subsubsection{Prädiktoren-Schülervariablen}

Schüleraspiration Klasse 6 Die Schüleraspiration wurde zum ersten Messzeitpunkt mit dem Item „Was möchtest du nach der Orientierungsstufe machen?“ erfasst. Orientierungsstufe bezeichnet in der Schweiz die Sekundarstufe I. Erneut verwendeten wir für die Operationalisierung die ISCED der UNESCO (2012; EDK 2015a). Dazu transformierten wir fünf Antwortkategorien (,eine ungelernte Arbeitstätigkeit“, „eine Anlehre“,,eine Berufslehre oder Berufsschule“, „eine Berufslehre mit Berufsmatura“, und „eine gymnasiale Matura“) in eine Dummyvariable $(0=$, ,berufsbildend “; $1=$,, allgemeinbildend“). Beispielsweise wurden Schüler/-innen, die eine Berufslehre machen wollten eine 0 und Schüler/-innen, die eine Matura aspirierten eine 1 zugewiesen. Die Schüleraspirationen korrelieren mittelstark mit der Elternaspirationen $(r=0,50)$ - unter Berücksichtigung der gesamten Stichprobe.

Prüfungsleistung Klasse 6 Am Ende der Grundschule mussten die Schüler/-innen verpflichtend einen standardisierten Leistungstest absolvieren, der aus einem Mathematik- und einem Deutschtest bestand. In jedem Testteil konnten maximal 50 Punkte erreicht werden, sodass insgesamt maximal 100 Punkte erzielt werden konnten. 
Notenschnitt Klasse 9 Diese Variable wird sowohl als Prädiktor, aber auch als Kriterium verwendet (siehe oben, „Abhängige Variablen“).

\subsubsection{Prädiktoren-Lehrervariablen}

Lehrerempfehlung Auf einer 5-stufigen Skala gaben die Lehrkräfte eine Zuweisungsempfehlung nach der Grundschule ab (5=,Gymnasium empfohlen “; $4=$, ,Gymnasium bedingt empfohlen “; 3 = ,, Realschule empfohlen “; 2 = „, Realschule bedingt empfohlen“; 1=,Hauptschule empfohlen“). Diese Angabe wurde zum ersten Messzeitpunkt erfasst.

\subsection{Statistische Analyse}

Die statistische Analyse erfolgte in mehreren Schritten und wurde mit dem Statistikprogramm Mplus 7.3 (Muthén und Muthén 1998-2012) durchgeführt. Zunächst wurden deskriptive Befunde für die Untersuchungsvariablen betrachtet. Anschließend wurden ausgehend vom Forschungsvorhaben verschiedene (logistische) Regressionsmodelle (z.B. Agresti 2002) berechnet. Zur Vorhersage der Korrekturen wurden die Gruppe der Realschüler/-innen und der Gymnasiasten getrennt untersucht; dies ist dadurch bedingt, dass die Schüler/-innen von der Realschule ausschließlich die Möglichkeit haben aufwärts zu korrigieren (Nachholen der Hochschulreife) und die Gymnasiasten dagegen ausschließlich abwärts korrigieren können (vorzeitiges Verlassen des Weges zur Hochschulreife). In der vorliegenden Arbeit wurden fehlende Werte (Enders 2001) mit dem in Mplus implementierten Full Information Maximum Likelihood (FIML)-Methode berücksichtigt. Des Weiteren wurde für die genestete Datenstruktur (Schüler/-innen in Klassen) der Standardfehler korrigiert (McNeish et al. 2017). Alle metrischen Variablen wurden z-standardisiert.

Interpretation der Ergebnisse Regressionskoeffizienten aus logistischen Regressionsanalysen sind schwer zu interpretieren. Häufig werden daher Odds Ratios (OR) berichtet, die jedoch nach Mood (2010) grundsätzlich und nach Kuha und Mills (2018) in einem bestimmten Fall als problematisch eingestuft werden. So sind Odds Ratios beispielsweise nicht vergleichbar zwischen Modellen mit unterschiedlichen unabhängigen Variablen (siehe Mood 2010). In der vorliegenden Studie werden daher neben den oft üblichen ORs auch die durchschnittlichen additiven Effekte der unabhängigen Variable auf die Wahrscheinlichkeit (Average Marginal Effect; AME) berichtet, die jedoch auch nicht frei von jeglicher Kritik sind (Williams 2015): „Wenn $\mathrm{x}_{\mathrm{i}}$ um eine Einheit steigt, steigt die Wahrscheinlichkeit $\mathrm{y}=1$ durchschnittlich um AME Punkte“ (Best und Wolf 2012, S. 383). Es sei darauf hingewiesen, dass bei diesen Durchschnittseffekten der nicht-lineare Verlauf der Wahrscheinlichkeitskurve ignoriert wird. 
Tab. 2 Nutzung der vertikalen Öffnung

\begin{tabular}{|c|c|c|c|c|c|c|}
\hline & \multicolumn{2}{|c|}{$\begin{array}{l}\text { Beabsichtigte Entscheidung } \\
\text { nach der Sek. I (Kl. 9) }\end{array}$} & \multirow[b]{2}{*}{ Gesamt } & \multicolumn{2}{|c|}{$\begin{array}{l}\text { Tatsächliche Entscheidung } \\
\text { nach der Sek. I (K1. 10) }\end{array}$} & \multirow[b]{2}{*}{ Gesamt } \\
\hline & $\begin{array}{l}\text { Keine } \\
\text { Korrektur }\end{array}$ & Korrektur & & $\begin{array}{l}\text { Keine } \\
\text { Korrektur }\end{array}$ & Korrektur & \\
\hline \multicolumn{7}{|c|}{ Schultyp am Ende der Sek. I } \\
\hline Realschule & $\begin{array}{l}358 \\
(57 \%)\end{array}$ & $\begin{array}{l}268 \\
(43 \%)\end{array}$ & $\begin{array}{l}626 \\
(100 \%)\end{array}$ & $\begin{array}{l}225 \\
(71 \%)\end{array}$ & $\begin{array}{l}90 \\
(29 \%)\end{array}$ & $\begin{array}{l}315 \\
(100 \%)\end{array}$ \\
\hline Gymnasium & $\begin{array}{l}326 \\
(77 \%)\end{array}$ & $\begin{array}{l}99 \\
(23 \%)\end{array}$ & $\begin{array}{l}425 \\
(100 \%)\end{array}$ & $\begin{array}{l}113 \\
(60 \%)\end{array}$ & $\begin{array}{l}74 \\
(40 \%)\end{array}$ & $\begin{array}{l}187 \\
(100 \%)\end{array}$ \\
\hline Gesamt & $\begin{array}{l}684 \\
(65 \%)\end{array}$ & $\begin{array}{l}367 \\
(35 \%)\end{array}$ & $\begin{array}{l}1051 \\
(100 \%)\end{array}$ & $\begin{array}{l}338 \\
(67 \%)\end{array}$ & $\begin{array}{l}164 \\
(33 \%)\end{array}$ & $\begin{array}{l}502 \\
(100 \%)\end{array}$ \\
\hline
\end{tabular}

Kreuztabelle zwischen den Schultypen am Ende der Sekundarstufe I (Sek. I) und den (beabsichtigten) Entscheidungen nach der Sek. I

\section{Ergebnisse}

\subsection{Deskriptive Befunde zur vertikalen Öffnung}

Befunde zur Nutzung der vertikalen Öffnung werden in Tab. 2 berichtet. Insgesamt lässt sich feststellen, dass $29 \%$ der Realschüler/-innen direkt nach dem Realschulabschluss den Weg zur Hochschulreife einschlugen und somit die Zuweisung nach der Grundschule „korrigierten“. Betrachtet man die Gymnasiasten, verließen $40 \%$ der Schüler/-innen den direkten Weg zur Hochschulreife. Demnach ist festzuhalten, dass im vorliegenden Bildungssystem mehr Schüler/-innen eine Abwärts- statt eine Aufwärtskorrektur vornahmen.

\subsection{Vorhersage der Zuweisung und des Schulerfolgs}

Nicht alle Schüler/-innen erhielten bereits nach der Grundschule die Möglichkeit ihre Aspirationen umzusetzen. So wurden $30 \%$ der Schüler/-innen zur Realschule und knapp 10\% zum Gymnasium zugewiesen, obwohl sie abweichende Aspirationen hatten. Die Vorhersagemodelle für die Zuweisung nach der Grundschule und den Schulerfolg in der Sekundarstufe I sind in Tab. 3 abgebildet. Zunächst testeten wir jeweils den Einfluss der Zuweisungselemente des Schulsystems in Deutschfreiburg - unter Kontrolle des sozialen Hintergrunds (Modell 1) auf die Zuweisung und den Schulerfolg. Anschließend inkludierten wir die Aspirationen der Eltern und Kinder (Modell 2).

Zuweisung Ob Schüler/-innen nach der Grundschule auf das Gymnasium wechselten, wurde durch die Lehrerempfehlung (Modell 2: $\mathrm{OR}=33,69$; $\mathrm{AME}=0,32$ ) und die Vergleichsprüfung (Modell 2: $\mathrm{OR}=26,18 ; \mathrm{AME}=0,11$ ) vorhergesagt - so wie es das Schulsystem in Deutschfreiburg vorsieht. Dementsprechend führten höhere Empfehlungen und bessere Leistungen zu einem höheren Schultyp der Sekundarstufe I. Darüber hinaus zeigte aber auch der HISEI-Wert der Familie einen kleinen 
Tab. 3 Vorhersagemodelle für die Zuweisung nach der Grundschule und den Schulerfolg in der Sekundarstufe I

\begin{tabular}{|c|c|c|c|c|c|c|}
\hline & \multicolumn{4}{|c|}{ Zuweisung (Gymnasium) } & \multicolumn{2}{|c|}{ Notenschnitt Klasse 9} \\
\hline & \multicolumn{2}{|l|}{ Modell 1} & \multicolumn{2}{|l|}{ Modell 2} & \multirow{2}{*}{$\begin{array}{l}\text { Modell } 1 \\
\beta \\
{[95 \% \mathrm{KI}]}\end{array}$} & \multirow{2}{*}{$\begin{array}{l}\text { Modell } 2 \\
\beta \\
{[95 \% \mathrm{KI}}\end{array}$} \\
\hline & $\begin{array}{l}\text { OR } \\
{[95 \% \mathrm{KI}]}\end{array}$ & $\begin{array}{l}\text { AME } \\
{[95 \% \mathrm{KI}]}\end{array}$ & $\begin{array}{l}\text { OR } \\
{[95 \% \mathrm{KI}]}\end{array}$ & $\begin{array}{l}\text { AME } \\
{[95 \% \mathrm{KI}]}\end{array}$ & & \\
\hline \multicolumn{7}{|c|}{ Lehrervariable } \\
\hline Empfehlung & $\begin{array}{l}33,62 \\
{[17,31 ;} \\
65,30]\end{array}$ & $\begin{array}{l}0,32 \\
{[0,26 ;} \\
0,37]\end{array}$ & $\begin{array}{l}33,69 \\
{[17,29 ;} \\
65,64]\end{array}$ & $\begin{array}{l}\mathbf{0 , 3 2} \\
{[0,27 ;} \\
0,37]\end{array}$ & $\begin{array}{l}0,34 \\
{[0,19 ;} \\
0,49]\end{array}$ & $\begin{array}{l}\mathbf{0 , 3 5} \\
{[\mathbf{0 , 1 9} ;} \\
\mathbf{0 , 5 0 ]}\end{array}$ \\
\hline \multicolumn{7}{|c|}{ Schülervariablen } \\
\hline $\begin{array}{l}\text { Prüfungs- } \\
\text { leistung } \\
\text { K1. } 6\end{array}$ & $\begin{array}{l}26,40 \\
{[13,36 ;} \\
52,18]\end{array}$ & $\begin{array}{l}0,11 \\
{[0,09 ;} \\
0,14]\end{array}$ & $\begin{array}{l}26,18 \\
{[13,27 ;} \\
51,64]\end{array}$ & $\begin{array}{l}0,11 \\
{[0,09 ;} \\
0,14]\end{array}$ & $\begin{array}{l}0,40 \\
{[0,33 ;} \\
0,47]\end{array}$ & $\begin{array}{l}0,39 \\
{[0,32 ;} \\
0,46]\end{array}$ \\
\hline $\begin{array}{l}\text { Aspiration } \\
\text { Kl. } 6\end{array}$ & - & - & $\begin{array}{l}1,19 \\
{[0,42 ;} \\
3,37]\end{array}$ & $\begin{array}{l}0,00 \\
{[-0,02} \\
0,03]\end{array}$ & - & $\begin{array}{l}\mathbf{0 , 2 1} \\
{[0,05 ;} \\
0,37]\end{array}$ \\
\hline \multicolumn{7}{|c|}{ Elternvariablen } \\
\hline Empfehlung & $\begin{array}{l}1,36 \\
{[0,75 ;} \\
2,47]\end{array}$ & $\begin{array}{l}0,04 * \\
{[0,00 ;} \\
0,08]\end{array}$ & $\begin{array}{l}1,35 \\
{[0,73 ;} \\
2,52]\end{array}$ & $\begin{array}{l}0,03 \\
{[-0,01} \\
0,07]\end{array}$ & $\begin{array}{l}0,07 \\
{[-0,06} \\
0,20]\end{array}$ & $\begin{array}{l}0,03 \\
{[-0,11 ;} \\
0,17]\end{array}$ \\
\hline HISEI & $\begin{array}{l}1,87 \\
{[1,20 ;} \\
2,91]\end{array}$ & $\begin{array}{l}\mathbf{0 , 0 2} \\
{[\mathbf{0 , 0 0} ;} \\
\mathbf{0 , 0 3}]\end{array}$ & $\begin{array}{l}1,86 \\
{[1,22 ;} \\
2,83]\end{array}$ & $\begin{array}{l}\mathbf{0 , 0 2} \\
{[\mathbf{0 , 0 0} ;} \\
\mathbf{0 , 0 3}]\end{array}$ & $\begin{array}{l}0,02 \\
{[-0,04} \\
0,09]\end{array}$ & $\begin{array}{l}0,01 \\
{[-0,06} \\
0,07]\end{array}$ \\
\hline $\begin{array}{l}\text { Bildungs- } \\
\text { abschluss }\end{array}$ & $\begin{array}{l}1,30 \\
{[0,58 ;} \\
2,91]\end{array}$ & $\begin{array}{l}0,00 \\
{[-0,03} \\
0,03]\end{array}$ & $\begin{array}{l}1,28 \\
{[0,57 ;} \\
2,88]\end{array}$ & $\begin{array}{l}0,00 \\
{[-0,03} \\
0,03]\end{array}$ & $\begin{array}{l}\mathbf{0 , 1 4} \\
{[0,01 ;} \\
\mathbf{0 , 2 6 ]}\end{array}$ & $\begin{array}{l}0,11 * \\
{[-0,01} \\
0,23]\end{array}$ \\
\hline $\begin{array}{l}\text { Sprach- } \\
\text { gebrauch }\end{array}$ & $\begin{array}{l}0,89 \\
{[0,29 ;} \\
2,77]\end{array}$ & $\begin{array}{l}0,01 \\
{[-0,02} \\
0,05]\end{array}$ & $\begin{array}{l}0,93 \\
{[0,29 ;} \\
2,91]\end{array}$ & $\begin{array}{l}0,01 \\
{[-0,03 ;} \\
0,05]\end{array}$ & $\begin{array}{l}-0,04 \\
{[-0,28} \\
0,20]\end{array}$ & $\begin{array}{l}0,01 \\
{[-0,23 ;} \\
0,24]\end{array}$ \\
\hline $\begin{array}{l}\text { Aspiration } \\
\text { K1. } 6\end{array}$ & - & - & $\begin{array}{l}0,92 \\
{[0,39 ;} \\
2,16]\end{array}$ & $\begin{array}{l}0,00 \\
{[-0,03} \\
0,03]\end{array}$ & - & $\begin{array}{l}0,03 \\
{[-0,11} \\
0,17]\end{array}$ \\
\hline Zuweisung & - & - & - & - & $\begin{array}{l}-\mathbf{0 , 9 6} \\
{[-1,21} \\
-0,72]\end{array}$ & $\begin{array}{c}-\mathbf{0 , 9 6} \\
{[-\mathbf{1 , 2 0}} \\
-\mathbf{0 , 7 2}]\end{array}$ \\
\hline (Pseudo) $-\mathrm{R}^{2}$ & 0,93 & 0,79 & 0,93 & 0,79 & 0,17 & 0,18 \\
\hline
\end{tabular}

$N=1219$ Realschüler/-innen und Gymnasiasten berücksichtigt

Fettgedruckte Koeffizienten sind signifikant ( $p<0,05$; zweiseitig); $*=p<0,05$; einseitig OR Odds Ratio, AME Average Marginal Effect, $\beta$ Regressionskoeffizient, HISEI Höchster sozioökonomischer Status, 95\% KI 95\%-iges Konfidenzintervall

signifikanten Effekt. Sowohl die Aspirationen der Kinder als auch der Eltern (gemessen in Klasse 6) kamen beim Übergang nach der Grundschule nicht zum Tragen.

Schulerfolg Von den Zuweisungselementen zeigten sowohl die Prüfungsleistung aus der Vergleichsprüfung (Modell 1: $\beta=0,40$ ) als auch die Lehrerempfehlung (Modell 1: $\beta=0,34$ ) eine prädiktive Kraft für den Notenschnitt in der neunten Klasse. Wurden die Aspirationen der Eltern und Kinder nicht berücksichtigt, beeinflusste der Bildungsabschluss der Eltern die Leistung innerhalb der Sekundarstufe I (Mo- 
Tab. 4 Vorhersage der beabsichtigten Aufwärtskorrektur am Ende der Sekundarstufe I

\begin{tabular}{|c|c|c|c|c|c|c|}
\hline & \multicolumn{2}{|l|}{ Modell 1} & \multicolumn{2}{|l|}{ Modell 2} & \multicolumn{2}{|l|}{ Modell 3} \\
\hline & $\begin{array}{l}\text { OR } \\
{[95 \% \mathrm{KI}]}\end{array}$ & $\begin{array}{l}\text { AME } \\
{[95 \% \mathrm{KI}]}\end{array}$ & $\begin{array}{l}\text { OR } \\
{[95 \% \mathrm{KI}]}\end{array}$ & $\begin{array}{l}\text { AME } \\
{[95 \% \mathrm{KI}]}\end{array}$ & $\begin{array}{l}\text { OR } \\
{[95 \% \mathrm{KI}]}\end{array}$ & $\begin{array}{l}\text { AME } \\
{[95 \% \mathrm{KI}]}\end{array}$ \\
\hline \multicolumn{7}{|l|}{ Lehrervariable } \\
\hline Empfehlung & $\begin{array}{l}1,01 \\
{[0,81 ;} \\
1,27]\end{array}$ & $\begin{array}{l}0,00 \\
{[-0,05} \\
0,04]\end{array}$ & $\begin{array}{l}0,99 \\
{[0,78 ;} \\
1,26]\end{array}$ & $\begin{array}{l}0,00 \\
{[-0,05} \\
0,04]\end{array}$ & $\begin{array}{l}0,88 \\
{[0,67 ;} \\
1,16]\end{array}$ & $\begin{array}{l}-0,02 \\
{[-0,06} \\
0,02]\end{array}$ \\
\hline \multicolumn{7}{|l|}{ Schülervariablen } \\
\hline $\begin{array}{l}\text { Prüfungsleistung } \\
\text { K1. } 6\end{array}$ & $\begin{array}{l}1,19 * \\
{[1,00 ;} \\
1,41]\end{array}$ & $\begin{array}{l}0,03 * \\
{[0,00 ;} \\
0,07]\end{array}$ & $\begin{array}{l}1,15 \\
{[0,97 ;} \\
1,36]\end{array}$ & $\begin{array}{l}0,03 \\
{[-0,01 ;} \\
0,06]\end{array}$ & $\begin{array}{l}0,89 \\
{[0,72 ;} \\
1,10]\end{array}$ & $\begin{array}{l}-0,02 \\
{[-0,06} \\
0,02]\end{array}$ \\
\hline Aspiration Kl. 6 & - & - & $\begin{array}{l}2,22 \\
{[1,42 ;} \\
1,47]\end{array}$ & $\begin{array}{l}0,18 \\
{[0,08 ;} \\
0,28]\end{array}$ & $\begin{array}{l}2,05 \\
{[1,24 ;} \\
3,38]\end{array}$ & $\begin{array}{l}0,14 \\
{[0,04 ;} \\
0,24]\end{array}$ \\
\hline $\begin{array}{l}\text { Notenschnitt } \\
\text { Kl. } 9\end{array}$ & - & - & - & - & $\begin{array}{l}2,56 \\
{[1,99 ;} \\
3,35]\end{array}$ & $\begin{array}{l}0,16 \\
{[0,13 ;} \\
0,20]\end{array}$ \\
\hline \multicolumn{7}{|l|}{ Elternvariablen } \\
\hline Empfehlung & $\begin{array}{l}1,48 \\
{[1,16 ;} \\
1,89]\end{array}$ & $\begin{array}{l}\mathbf{0 , 0 8} \\
{[0,04 ;} \\
0,13]\end{array}$ & $\begin{array}{l}1,27 * \\
{[0,97 ;} \\
1,66]\end{array}$ & $\begin{array}{l}0,05^{*} \\
{[0,00 ;} \\
0,10]\end{array}$ & $\begin{array}{l}1,26 \\
{[0,93 ;} \\
1,71]\end{array}$ & $\begin{array}{l}0,04 \\
{[-0,01} \\
0,09]\end{array}$ \\
\hline HISEI & $\begin{array}{l}1,48 \\
{[1,20 ;} \\
1,83]\end{array}$ & $\begin{array}{l}\mathbf{0 , 0 8} \\
{[\mathbf{0 , 0 4} ;} \\
\mathbf{0 , 1 3}]\end{array}$ & $\begin{array}{l}1,39 \\
{[1,10 ;} \\
1,75]\end{array}$ & $\begin{array}{l}\mathbf{0 , 0 6} \\
{[0,02 ;} \\
0,11]\end{array}$ & $\begin{array}{l}1,47 \\
{[1,15 ;} \\
1,89]\end{array}$ & $\begin{array}{l}\mathbf{0 , 0 7} \\
{[\mathbf{0 , 0 2} ;} \\
\mathbf{0 , 1 1}]\end{array}$ \\
\hline Bildungsabschluss & $\begin{array}{l}1,46 \\
{[0,93 ;} \\
2,29]\end{array}$ & $\begin{array}{l}0,08 \\
{[-0,02} \\
0,18]\end{array}$ & $\begin{array}{l}1,24 \\
{[0,76 ;} \\
2,01]\end{array}$ & $\begin{array}{l}0,05 \\
{[-0,05} \\
0,15]\end{array}$ & $\begin{array}{l}1,17 \\
{[0,69 ;} \\
2,01]\end{array}$ & $\begin{array}{l}0,03 \\
{[-0,07} \\
0,12]\end{array}$ \\
\hline Sprachgebrauch & $\begin{array}{l}0,29 \\
{[0,16 ;} \\
0,51]\end{array}$ & $\begin{array}{l}-0,26 \\
{[-0,37} \\
-0,15]\end{array}$ & $\begin{array}{l}0,36 \\
{[0,19 ;} \\
0,69]\end{array}$ & $\begin{array}{l}-\mathbf{0 , 2 0} \\
{[-\mathbf{0 , 3 2}} \\
-\mathbf{0 , 0 8}]\end{array}$ & $\begin{array}{l}0,32 \\
{[0,17 ;} \\
0,63]\end{array}$ & $\begin{array}{l}-\mathbf{0 , 2 0} \\
{[-\mathbf{0 , 3 1}} \\
-\mathbf{0 , 0 8}]\end{array}$ \\
\hline Aspiration Kl. 6 & - & - & $\begin{array}{l}2,26 \\
{[1,39 ;} \\
3,66]\end{array}$ & $\begin{array}{l}\mathbf{0 , 1 8} \\
{[0,07 ;} \\
0,28]\end{array}$ & $\begin{array}{l}2,69 \\
{[1,59 ;} \\
4,57]\end{array}$ & $\begin{array}{l}\mathbf{0 , 1 8} \\
{[0,08 ;} \\
0,28]\end{array}$ \\
\hline$($ Pseudo $)-\mathrm{R}^{2}$ & 0,18 & $\mathbf{0 , 1 3}$ & 0,26 & $\mathbf{0 , 2 0}$ & 0,41 & 0,29 \\
\hline
\end{tabular}

$N=626$ Realschüler/-innen

Fettgedruckte Koeffizienten sind signifikant ( $p<0,05$; zweiseitig); $*=p<0,05$; einseitig

$O R$ Odds Ratio, AME Average Marginal Effect, HISEI Höchster sozioökonomischer Status, 95\% KI 95\%-iges Konfidenzintervall

dell 1, $\beta=0,14)$, während sich dieser Effekt in Modell 2 nicht mehr zeigte. Jedoch beeinflussten die Schüleraspirationen am Ende der Grundschule (Klasse 6; $\beta=0,21$ ) den Schulerfolg - unter Kontrolle aller anderen Prädiktoren. Je höher die Aspirationen der Schüler/-innen am Ende der Grundschule waren, desto besser war ihr Notenschnitt am Ende der Sekundarstufe I. Die elterlichen Aspirationen am Ende der Grundschule (Klasse 6) hatten keine statistisch signifikante Vorhersageleistung. 
Tab. 5 Vorhersage der tatsächlichen Aufwärtskorrektur am Ende der Sekundarstufe I

\begin{tabular}{|c|c|c|c|c|c|c|}
\hline & \multicolumn{2}{|l|}{ Modell 1} & \multicolumn{2}{|l|}{ Modell 2} & \multicolumn{2}{|l|}{ Modell 3} \\
\hline & $\begin{array}{l}\text { OR } \\
{[95 \% \mathrm{KI}]}\end{array}$ & $\begin{array}{l}\text { AME } \\
{[95 \% \mathrm{KI}]}\end{array}$ & $\begin{array}{l}\text { OR } \\
{[95 \% \mathrm{KI}]}\end{array}$ & $\begin{array}{l}\text { AME } \\
{[95 \% \mathrm{KI}]}\end{array}$ & $\begin{array}{l}\text { OR } \\
{[95 \% \mathrm{KI}]}\end{array}$ & $\begin{array}{l}\text { AME } \\
{[95 \% \mathrm{KI}]}\end{array}$ \\
\hline \multicolumn{7}{|l|}{ Lehrervariable } \\
\hline Empfehlung & $\begin{array}{l}1,15 \\
{[0,78 ;} \\
1,69]\end{array}$ & $\begin{array}{l}0,02 \\
{[-0,05} \\
0,09]\end{array}$ & $\begin{array}{l}1,07 \\
{[0,68 ;} \\
1,69]\end{array}$ & $\begin{array}{l}0,01 \\
{[-0,06} \\
0,09]\end{array}$ & $\begin{array}{l}0,93 \\
{[0,56 ;} \\
1,55]\end{array}$ & $\begin{array}{l}-0,01 \\
{[-0,08} \\
0,07]\end{array}$ \\
\hline \multicolumn{7}{|l|}{ Schülervariablen } \\
\hline $\begin{array}{l}\text { Prüfungsleistung } \\
\text { K1. } 6\end{array}$ & $\begin{array}{l}1,23 \\
{[0,95 ;} \\
1,60]\end{array}$ & $\begin{array}{l}0,04 \\
{[-0,01} \\
0,08]\end{array}$ & $\begin{array}{l}1,13 \\
{[0,88 ;} \\
1,45]\end{array}$ & $\begin{array}{l}0,02 \\
{[-0,02} \\
0,06]\end{array}$ & $\begin{array}{l}0,89 \\
{[0,70 ;} \\
1,19]\end{array}$ & $\begin{array}{l}-0,02 \\
{[-0,06} \\
0,02]\end{array}$ \\
\hline Aspiration Kl. 6 & - & - & $\begin{array}{l}2,54 \\
{[1,20 ;} \\
5,38]\end{array}$ & $\begin{array}{l}0,15 \\
{[0,03 ;} \\
0,27]\end{array}$ & $\begin{array}{l}3,20 \\
{[1,40 ;} \\
7,35]\end{array}$ & $\begin{array}{l}\mathbf{0 , 1 5} \\
{[0,04 ;} \\
0,27]\end{array}$ \\
\hline $\begin{array}{l}\text { Notenschnitt } \\
\text { Kl. } 9\end{array}$ & - & - & - & - & $\begin{array}{l}3,50 \\
{[2,35 ;} \\
5,21]\end{array}$ & $\begin{array}{l}\mathbf{0 , 1 7} \\
{[0,13 ;} \\
0,21]\end{array}$ \\
\hline \multicolumn{7}{|l|}{ Elternvariablen } \\
\hline Empfehlung & $\begin{array}{l}1,22 \\
{[0,91 ;} \\
1,63]\end{array}$ & $\begin{array}{l}0,04 \\
{[-0,02} \\
0,10]\end{array}$ & $\begin{array}{l}0,92 \\
{[0,63 ;} \\
1,35]\end{array}$ & $\begin{array}{l}-0,02 \\
{[-0,08} \\
0,05]\end{array}$ & $\begin{array}{l}0,83 \\
{[0,52 ;} \\
1,30]\end{array}$ & $\begin{array}{l}-0,02 \\
{[-0,09} \\
0,04]\end{array}$ \\
\hline HISEI & $\begin{array}{l}1,44 * \\
{[0,99 ;} \\
2,07]\end{array}$ & $\begin{array}{l}0,07 * \\
{[0,00 ;} \\
0,13]\end{array}$ & $\begin{array}{l}1,39 \\
{[0,94 ;} \\
2,05]\end{array}$ & $\begin{array}{l}0,05 \\
{[-0,01} \\
0,11]\end{array}$ & $\begin{array}{l}1,39 \\
{[0,94 ;} \\
2,04]\end{array}$ & $\begin{array}{l}0,04 \\
{[-0,01 ;} \\
0,09]\end{array}$ \\
\hline Bildungsabschluss & $\begin{array}{l}1,72 \\
{[0,89 ;} \\
3,30]\end{array}$ & $\begin{array}{l}0,10 * \\
{[-0,01} \\
0,21]\end{array}$ & $\begin{array}{l}1,28 \\
{[0,63 ;} \\
2,59]\end{array}$ & $\begin{array}{l}0,05 \\
{[-0,06} \\
0,15]\end{array}$ & $\begin{array}{l}1,19 \\
{[0,56 ;} \\
2,53]\end{array}$ & $\begin{array}{l}0,03 \\
{[-0,07} \\
0,12]\end{array}$ \\
\hline Sprachgebrauch & $\begin{array}{l}0,53 \\
{[0,25 ;} \\
1,11]\end{array}$ & $\begin{array}{l}-0,13 \\
{[-0,29} \\
0,03]\end{array}$ & $\begin{array}{l}0,73 \\
{[0,30 ;} \\
1,76]\end{array}$ & $\begin{array}{l}-0,06 \\
{[-0,23} \\
0,11]\end{array}$ & $\begin{array}{l}0,42 \\
{[0,17 ;} \\
1,07]\end{array}$ & $\begin{array}{l}-0,11 \\
{[-0,26} \\
0,05]\end{array}$ \\
\hline Aspiration Kl. 6 & - & - & $\begin{array}{l}3,48 \\
{[1,83 ;} \\
6,61]\end{array}$ & $\begin{array}{l}0,28 \\
{[0,14 ;} \\
0,40]\end{array}$ & $\begin{array}{l}4,60 \\
{[2,05 ;} \\
10,29]\end{array}$ & $\begin{array}{l}0,28 \\
{[0,15 ;} \\
0,41]\end{array}$ \\
\hline$($ Pseudo $)-\mathrm{R}^{2}$ & 0,16 & $\mathbf{0 , 1 0}$ & 0,26 & $\mathbf{0 , 2 0}$ & 0,48 & $\mathbf{0 , 3 2}$ \\
\hline
\end{tabular}

$N=315$ Realschüler/-innen

Fettgedruckte Koeffizienten sind signifikant ( $p<0,05$; zweiseitig); $*=p<0,05$; einseitig

$O R$ Odds Ratio, AME Average Marginal Effect, HISEI Höchster sozioökonomischer Status, 95\% KI 95\%-iges Konfidenzintervall

\subsection{Vorhersagen der Auf- und Abwärtskorrekturen nach der Sekundarstufe I}

Aufwärtskorrektur Von den Realschüler/-innen, die bereits in der Grundschule höhere Aspirationen aufwiesen, schlugen $45 \%$ nach der Sekundarstufe I den Weg zur Hochschulreife ein. Wir spezifizierten für die beabsichtigte und tatsächliche Aufwärtskorrektur jeweils drei Vorhersagemodelle. Modell 1 berücksichtigte die Zuweisungselemente der Grundschule und die Variablen des sozialen Hintergrunds, Modell 2 umfasste zusätzlich die Aspirationen der Eltern und Kinder und Modell 3 inkludierte darüber hinaus die Leistung am Ende der Sekundarstufe I. 
Tab. 6 Vorhersage der beabsichtigten Abwärtskorrektur am Ende der Sekundarstufe I

\begin{tabular}{|c|c|c|c|c|c|c|}
\hline & \multicolumn{2}{|l|}{ Modell 1} & \multicolumn{2}{|l|}{ Modell 2} & \multicolumn{2}{|l|}{ Modell 3} \\
\hline & $\begin{array}{l}\text { OR } \\
{[95 \% \mathrm{KI}]}\end{array}$ & $\begin{array}{l}\text { AME } \\
{[95 \% \mathrm{KI}]}\end{array}$ & $\begin{array}{l}\text { OR } \\
{[95 \% \mathrm{KI}]}\end{array}$ & $\begin{array}{l}\text { AME } \\
{[95 \% \mathrm{KI}]}\end{array}$ & $\begin{array}{l}\text { OR } \\
{[95 \% \mathrm{KI}]}\end{array}$ & $\begin{array}{l}\text { AME } \\
{[95 \% \mathrm{KI}]}\end{array}$ \\
\hline \multicolumn{7}{|l|}{ Lehrervariable } \\
\hline Empfehlung & $\begin{array}{l}1,07 \\
{[0,69 ;} \\
1,66]\end{array}$ & $\begin{array}{l}0,01 \\
{[-0,06} \\
0,07]\end{array}$ & $\begin{array}{l}1,00 \\
{[0,66 ;} \\
1,52]\end{array}$ & $\begin{array}{l}-0,01 \\
{[-0,05} \\
0,05]\end{array}$ & $\begin{array}{l}1,18 \\
{[0,78 ;} \\
1,78]\end{array}$ & $\begin{array}{l}0,02 \\
{[-0,03} \\
0,07]\end{array}$ \\
\hline \multicolumn{7}{|l|}{ Schülervariablen } \\
\hline $\begin{array}{l}\text { Prüfungsleistung } \\
\text { K1. } 6\end{array}$ & $\begin{array}{l}0,99 \\
{[0,75 ;} \\
1,30]\end{array}$ & $\begin{array}{l}0,00 \\
{[-0,04} \\
0,04]\end{array}$ & $\begin{array}{l}1,02 \\
{[0,76 ;} \\
1,37]\end{array}$ & $\begin{array}{l}0,01 \\
{[-0,03 ;} \\
0,04]\end{array}$ & $\begin{array}{l}1,21 \\
{[0,89 ;} \\
1,66]\end{array}$ & $\begin{array}{l}0,03 \\
{[-0,01} \\
0,06]\end{array}$ \\
\hline $\begin{array}{l}\text { Aspiration } \\
\text { K1. } 6\end{array}$ & - & - & $\begin{array}{l}0,34 \\
{[0,17 ;} \\
0,68]\end{array}$ & $\begin{array}{l}-\mathbf{0 , 2 2} \\
{[-0,36 ;} \\
-\mathbf{0 , 0 8}]\end{array}$ & $\begin{array}{l}\mathbf{0 , 4 0} \\
{[0,20 ;} \\
0,81]\end{array}$ & $\begin{array}{l}-0,20 \\
{[-0,34 ;} \\
-0,05]\end{array}$ \\
\hline $\begin{array}{l}\text { Notenschnitt } \\
\text { Kl. } 9\end{array}$ & - & - & - & - & $\begin{array}{l}\mathbf{0 , 5 7} \\
{[\mathbf{0 , 4 0} ;} \\
\mathbf{0 , 8 4}]\end{array}$ & $\begin{array}{l}-\mathbf{0 , 0 7} \\
{[-\mathbf{0 , 1 2} ;} \\
-\mathbf{0 , 0 2}]\end{array}$ \\
\hline \multicolumn{7}{|l|}{ Elternvariablen } \\
\hline Empfehlung & $\begin{array}{l}0,69 * \\
{[0,45} \\
1,05]\end{array}$ & $\begin{array}{l}-0,06 * \\
{[-0,14} \\
0,01]\end{array}$ & $\begin{array}{l}0,78 \\
{[0,52 ;} \\
1,18]\end{array}$ & $\begin{array}{l}-0,04 \\
{[-0,10} \\
0,02]\end{array}$ & $\begin{array}{l}0,69 * \\
{[0,46 ;} \\
1,03]\end{array}$ & $\begin{array}{l}-0,05 \\
{[-0,11} \\
0,01]\end{array}$ \\
\hline HISEI & $\begin{array}{l}0,54 \\
{[0,40 ;} \\
0,71]\end{array}$ & $\begin{array}{l}-0,09 \\
{[-0,14 ;} \\
-0,05]\end{array}$ & $\begin{array}{l}0,63 \\
{[0,46 ;} \\
0,86]\end{array}$ & $\begin{array}{l}-\mathbf{0 , 0 6} \\
{[-\mathbf{0 , 1 0}} \\
-\mathbf{0 , 0 1}]\end{array}$ & $\begin{array}{l}0,62 \\
{[0,46 ;} \\
0,85]\end{array}$ & $\begin{array}{l}-0,06 \\
{[-0,10} \\
-0,02]\end{array}$ \\
\hline Bildungsabschluss & $\begin{array}{l}0,48 \\
{[0,27 ;} \\
0,87]\end{array}$ & $\begin{array}{l}-\mathbf{0 , 1 4} \\
{[-0,26 ;} \\
-0,02]\end{array}$ & $\begin{array}{l}0,62 \\
{[0,33 ;} \\
1,19]\end{array}$ & $\begin{array}{l}-0,08 \\
{[-0,20} \\
0,36]\end{array}$ & $\begin{array}{l}0,68 \\
{[0,34 ;} \\
1,37]\end{array}$ & $\begin{array}{l}-0,07 \\
{[-0,19} \\
0,05]\end{array}$ \\
\hline Sprachgebrauch & $\begin{array}{l}6,45 \\
{[1,96 ;} \\
22,22]\end{array}$ & $\begin{array}{l}\mathbf{0 , 1 9} \\
{[-\mathbf{0 , 1 0}} \\
\mathbf{0 , 2 9}]\end{array}$ & $\begin{array}{l}3,88 \\
{[0,97 ;} \\
15,52]\end{array}$ & $\begin{array}{l}0,12 \\
{[0,00 ;} \\
0,22]\end{array}$ & $\begin{array}{l}3,69 \\
{[0,96 ;} \\
14,17]\end{array}$ & $\begin{array}{l}\mathbf{0 , 1 1} \\
{[0,01 ;} \\
0,21]\end{array}$ \\
\hline $\begin{array}{l}\text { Aspiration } \\
\text { Kl. } 6\end{array}$ & - & - & $\begin{array}{l}0,35 \\
{[0,21 ;} \\
0,59]\end{array}$ & $\begin{array}{l}-\mathbf{0 , 1 6} \\
{[-0,25} \\
-0,08]\end{array}$ & $\begin{array}{l}0,36 \\
{[0,21 ;} \\
0,62]\end{array}$ & $\begin{array}{l}-0,15 \\
{[-0,24 ;} \\
-0,06]\end{array}$ \\
\hline (Pseudo) $-\mathrm{R}^{2}$ & 0,25 & 0,14 & 0,37 & $\mathbf{0 , 2 7}$ & 0,41 & 0,29 \\
\hline
\end{tabular}

$N=425$ Gymnasiasten

Fettgedruckte Koeffizienten sind signifikant $(p<0,05$; zweiseitig); $*=p<0,05$; einseitig

$O R$ Odds Ratio, AME Average Marginal Effect, HISEI Höchster sozioökonomischer Status, 95\% KI 95\%-iges Konfidenzintervall

Tab. 4 präsentiert die Befunde für die beabsichtigte und Tab. 5 für die tatsächliche Aufwärtskorrektur.

Beabsichtigte Entscheidung nach der Sekundarstufe I Zur Vorhersage der beabsichtigten Aufwärtskorrektur zeigten im ersten Modell die Elternempfehlung $(\mathrm{OR}=1,48$; $\mathrm{AME}=0,08)$, der HISEI-Wert $(\mathrm{OR}=1,48 ; \mathrm{AME}=0,08)$ und der Sprachgebrauch in der Familie $(\mathrm{OR}=0,29$; $\mathrm{AME}=-0,26)$ einen signifikanten $\mathrm{Zu}$ sammenhang. Nach Hinzunahme der Aspirationen war die Elternempfehlung nicht mehr signifikant. Es zeigte sich sowohl in Modell 2 als auch in Modell 3, dass die Aspirationen der Eltern (Modell 3: $\mathrm{OR}=2,69$; $\mathrm{AME}=0,18$ ) und Kinder (Modell 3: 
Tab. 7 Vorhersage der tatsächlichen Abwärtskorrektur am Ende der Sekundarstufe I

\begin{tabular}{|c|c|c|c|c|c|c|}
\hline & \multicolumn{2}{|l|}{ Modell 1} & \multicolumn{2}{|l|}{ Modell 2} & \multicolumn{2}{|l|}{ Modell 3} \\
\hline & $\begin{array}{l}\text { OR } \\
{[95 \% \mathrm{KI}]}\end{array}$ & $\begin{array}{l}\text { AME } \\
{[95 \%} \\
\mathrm{KI}]\end{array}$ & $\begin{array}{l}\text { OR } \\
{[95 \% \mathrm{KI}]}\end{array}$ & $\begin{array}{l}\text { AME } \\
{[95 \%} \\
\mathrm{KI}]\end{array}$ & $\begin{array}{l}\text { OR } \\
{[95 \% \mathrm{KI}]}\end{array}$ & $\begin{array}{l}\text { AME } \\
{[95 \%} \\
\mathrm{KI}]\end{array}$ \\
\hline \multicolumn{7}{|l|}{ Lehrervariable } \\
\hline Empfehlung & $\begin{array}{l}0,80 \\
{[0,39 ;} \\
1,63]\end{array}$ & $\begin{array}{l}-0,04 \\
{[-0,19 ;} \\
0,12]\end{array}$ & $\begin{array}{l}0,66 \\
{[0,34 ;} \\
1,30]\end{array}$ & $\begin{array}{l}-0,08 \\
{[-0,21 ;} \\
0,05]\end{array}$ & $\begin{array}{l}0,64 \\
{[0,32 ;} \\
1,28]\end{array}$ & $\begin{array}{l}-0,08 \\
{[-0,22 ;} \\
0,05]\end{array}$ \\
\hline \multicolumn{7}{|l|}{ Schülervariablen } \\
\hline $\begin{array}{l}\text { Prüfungsleistung } \\
\text { K1. } 6\end{array}$ & $\begin{array}{l}0,81 \\
{[0,57 ;} \\
1,15]\end{array}$ & $\begin{array}{l}-0,04 \\
{[-0,10 ;} \\
0,02]\end{array}$ & $\begin{array}{l}0,86 \\
{[0,60 ;} \\
1,25]\end{array}$ & $\begin{array}{l}-0,02 \\
{[-0,08 ;} \\
0,04]\end{array}$ & $\begin{array}{l}0,86 \\
{[0,58 ;} \\
1,27]\end{array}$ & $\begin{array}{l}-0,03 \\
{[-0,08} \\
0,04]\end{array}$ \\
\hline Aspiration Kl. 6 & - & - & $\begin{array}{l}\mathbf{0 , 1 7} \\
{[\mathbf{0 , 0 5} ;} \\
\mathbf{0 , 5 7}]\end{array}$ & $\begin{array}{l}-0,37 \\
{[-0,60 ;} \\
-0,14]\end{array}$ & $\begin{array}{l}\mathbf{0 , 1 5} \\
{[0,04 ;} \\
0,56]\end{array}$ & $\begin{array}{l}-0,38 \\
{[-0,61 ;} \\
-0,15]\end{array}$ \\
\hline $\begin{array}{l}\text { Notenschnitt } \\
\text { K1. } 9\end{array}$ & - & - & - & - & $\begin{array}{l}0,99 \\
{[0,59 ;} \\
1,64]\end{array}$ & $\begin{array}{l}0,00 \\
{[-0,08 ;} \\
0,08]\end{array}$ \\
\hline \multicolumn{7}{|l|}{ Elternvariablen } \\
\hline Empfehlung & $\begin{array}{l}0,98 \\
{[0,52 ;} \\
1,85]\end{array}$ & $\begin{array}{l}-0,01 \\
{[-0,15} \\
0,13]\end{array}$ & $\begin{array}{l}1,41 \\
{[0,80 ;} \\
2,48]\end{array}$ & $\begin{array}{l}0,07 \\
{[-0,04 ;} \\
0,19]\end{array}$ & $\begin{array}{l}1,45 \\
{[0,80 ;} \\
2,63]\end{array}$ & $\begin{array}{l}0,08 \\
{[-0,04 ;} \\
0,20]\end{array}$ \\
\hline HISEI & $\begin{array}{l}\mathbf{0 , 5 3} \\
{[\mathbf{0 , 3 5} ;} \\
\mathbf{0 , 8 2}]\end{array}$ & $\begin{array}{c}-\mathbf{0 , 1 2} \\
{[-\mathbf{0 , 1 9}} \\
-0,04]\end{array}$ & $\begin{array}{l}0,66^{*} \\
{[0,42 ;} \\
1,05]\end{array}$ & $\begin{array}{l}-0,06 \\
{[-0,14 ;} \\
0,02]\end{array}$ & $\begin{array}{l}0,67 * \\
{[0,43 ;} \\
1,05]\end{array}$ & $\begin{array}{l}-0,06 \\
{[-0,14 ;} \\
0,01]\end{array}$ \\
\hline Bildungsabschluss & $\begin{array}{l}\mathbf{0 , 3 8} \\
{[0,16 ;} \\
0,92]\end{array}$ & $\begin{array}{l}-0,22 \\
{[-0,41 ;} \\
-0,02]\end{array}$ & $\begin{array}{l}0,45 \\
{[0,17 ;} \\
1,22]\end{array}$ & $\begin{array}{l}-0,15 \\
{[-0,35} \\
0,05]\end{array}$ & $\begin{array}{l}0,47 \\
{[0,17 ;} \\
1,30]\end{array}$ & $\begin{array}{l}-0,15 \\
{[-0,34 ;} \\
0,06]\end{array}$ \\
\hline Sprachgebrauch & $\begin{array}{l}12,50 \\
{[3,58 ;} \\
43,67]\end{array}$ & $\begin{array}{l}\mathbf{0 , 3 4} \\
{[0,20 ;} \\
0,49]\end{array}$ & $\begin{array}{l}10,44 \\
{[2,24 ;} \\
48,57]\end{array}$ & $\begin{array}{l}0,28 \\
{[0,12 ;} \\
0,44]\end{array}$ & $\begin{array}{l}11,55 \\
{[2,67 ;} \\
44,92]\end{array}$ & $\begin{array}{l}0,29 \\
{[0,13 ;} \\
0,44]\end{array}$ \\
\hline Aspiration K1. 6 & - & - & $\begin{array}{l}0,58 * \\
{[0,33 ;} \\
1,01]\end{array}$ & $\begin{array}{l}-0,11 * \\
{[-0,22 ;} \\
0,00]\end{array}$ & $\begin{array}{l}0,59 * \\
{[0,35 ;} \\
1,01]\end{array}$ & $\begin{array}{l}-0,11 * \\
{[-0,21 ;} \\
0,01]\end{array}$ \\
\hline (Pseudo) $-\mathrm{R}^{2}$ & 0,29 & 0,19 & 0,38 & 0,28 & 0,38 & 0,28 \\
\hline
\end{tabular}

$N=187$ Gymnasiasten

Fettgedruckte Koeffizienten sind signifikant ( $p<0,05$; zweiseitig); $*=p<0,05$; einseitig

OR Odds Ratio, AME Average Marginal Effect, HISEI Höchster sozioökonomischer Status, 95\% KI 95\%-iges Konfidenzintervall

$\mathrm{OR}=2,05 ; \mathrm{AME}=0,14)$ aus der Grundschule die Aufwärtskorrektur vorhersagten - unter Kontrolle aller anderen Prädiktoren. Je höher die Aspirationen in der Grundschule waren, desto eher strebten die Schüler/-innen den direkten Weg zur Hochschulreife an.

Tatsächliche Entscheidung nach der Sekundarstufe I Es zeigte sich für die tatsächliche Entscheidung von Realschüler/-innen ein identisches Befundmuster hinsichtlich der Aspirationen der Eltern und Kinder im Vergleich zur beabsichtigten Übergangsentscheidung. Dementsprechend zeigten die Aspirationen von Schüler/ 
-innen $(\mathrm{OR}=3,20 ; \mathrm{AME}=0,15)$ und die der Eltern $(\mathrm{OR}=4,60 ; \mathrm{AME}=0,28)$ einen positiven Zusammenhang mit der Entscheidung (Modell 3). Je höher die Aspirationen von Kindern und Eltern in der Grundschule waren, desto höher war die Chance, dass die einstigen Realschüler/-innen sich für den Weg zur Hochschulreife entschieden. Die Zuweisungselemente nach der Grundschule und der soziale Hintergrund beeinflussten dagegen die tatsächliche Entscheidung nicht. Des Weiteren hatte die Leistung am Ende der Sekundarstufe I einen signifikanten Effekt $(\mathrm{OR}=3,50 ; \mathrm{AME}=0,17)$ auf die tatsächliche Entscheidung darüber, ob die Schüler/ innen auf den direkten Weg zur Hochschulreife wechselten.

Abwärtskorrektur $77 \%$ der Schüler/-innen vom Gymnasium, die in der Grundschule niedrigere Aspirationen aufwiesen, verließen nach der Sekundarstufe I den direkten Weg zur Hochschulreife. Wir testeten zur Vorhersage der beabsichtigten und tatsächlichen Abwärtskorrektur ebenfalls jeweils drei Modelle - dem vorherigen Vorgehen entsprechend.

Beabsichtigte Entscheidung nach der Sekundarstufe I Tab. 6 bildet die Befunde für die beabsichtigte Entscheidung ab. Neben dem Effekt des HISEI-Wertes $(\mathrm{OR}=0,62 ; \mathrm{AME}=-0,06)$ und der Leistung in der neunten Klasse $(\mathrm{OR}=0,57$; $\mathrm{AME}=-0,07)$, hatten die Aspirationen der Eltern $(\mathrm{OR}=0,36 ; \mathrm{AME}=-0,15)$ und der Kinder $(\mathrm{OR}=0,40$; $\mathrm{AME}=-0,20)$ eine prädiktive Kraft bei der beabsichtigten Entscheidung nach der Sekundarstufe I (Modell 3). Je niedriger die Aspirationen in der Grundschule waren, desto eher wollten Schüler/-innen den Weg zur Hochschulreife verlassen. Anders als bei der Vorhersage der beabsichtigten Aufwärtskorrektur, waren die Zuweisungselemente der Grundschule (Prüfungsleistung und Elternempfehlung) in keinem Modell signifikant.

Tatsächliche Entscheidung nach der Sekundarstufe I Tab. 7 präsentiert die Ergebnisse der logistischen Regressionsmodelle zur Vorhersage der tatsächlichen Abwärtskorrektur. Von den Schülervariablen wies lediglich die Aspiration eine Vorhersagekraft für die Entscheidung auf, ob jemand vorzeitig den Weg zur Hochschulreife verlässt (Modell 3: $\mathrm{OR}=0,15 ; \mathrm{AME}=-0,38$ ). Kinder, die bereits in der Grundschule niedrigere Aspirationen hatten, hatten eine höhere Chance den Weg zur Hochschulreife vorzeitig abzubrechen. Alle Zuweisungselemente des Übergangs in die Sekundarstufe I und auch die Leistung am Ende der Sekundarstufe hatten keine prädiktive Kraft. Auch die elterlichen Aspirationen hatten keinen Effekt auf die Vorhersage, ob Schüler/-innen den Weg zur Hochschulreife abbrachen. Neben den Aspirationen der Gymnasiasten übernahm nur noch der Sprachgebrauch in der Familie eine Vorhersagekraft $(\mathrm{OR}=11,55 ; \mathrm{AME}=0,29)$. Demnach hatten Kinder, deren Familien zu Hause Deutsch sprechen, eine höhere Chance den Weg zur Hochschulreife vorzeitig zu verlassen. 


\section{Diskussion}

In Deutschfreiburg stellen sowohl das Nachholen der Hochschulreife als auch das vorzeitige Verlassen des Weges zur Hochschulreife „Korrekturen von zugewiesenen Bildungswegen“ dar. Unklar war bisher jedoch, ob diese Auf- und Abwärtskorrekturen gleichzeitig das Resultat langgehegter Aspirationen darstellen.

\subsection{Zentrale Ergebnisse und Erklärungsansätze}

Einfluss der Aspirationen Mit Blick auf die erste Hypothese wurde gezeigt, dass das strenggeregelte Zuweisungsverfahren in Deutschfreiburg keinen Einfluss von Aspirationen beim Übertritt in die Sekundarstufe I zuließ. Jedoch hatten die Aspirationen, die Schüler/-innen bereits in der Grundschule (6. Klasse) besaßen, einen Einfluss auf den Schulerfolg (Hypothese 2) und alle Entscheidungen im Anschluss an die Sekundarstufe I (Hypothese 3). Ein ähnliches Befundmuster zeigte sich für die elterlichen Aspirationen (erfasst in der Grundschule, 6. Klasse). Sie wirkten sich ebenfalls auf die Aufwärtskorrektur und die beabsichtige Abwärtskorrektur aus. Jedoch beeinflussten die Elternaspirationen nicht das tatsächliche vorzeitige Verlassen des Weges zur Hochschulreife. Mögliche Erklärungen hierfür wären, dass Schüler/ -innen diese Entscheidung eigenständig treffen und Eltern so dem Wunsch des Kindes folgen (Wohlkinger und Ditton 2012) oder die Eltern beispielsweise die Leistung des Kindes überschätzten. Dass Kinder bereits in der Grundschule eigene konkrete Vorstellungen von ihren späteren Bildungswegen haben, konnten bereits Wohlkinger und Ditton (2012) aufzeigen. Des Weiteren sind die Ergebnisse der vorliegenden Studie im Einklang mit dem Modell der Wisconsin-Schule (Sewell et al. 1970), da Schüler/-innen und ihre Eltern anscheinend frühzeitig ihre Aspirationen festlegen und auf die Verwirklichung dieser warten (vgl. Kleine et al. 2009). Dennoch sei noch einmal darauf hingewiesen, dass andere Theorien bzw. Modelle davon ausgehen, dass sich Aspirationen ändern können; in der vorliegenden Studie wurde jedoch nicht die Veränderung von Aspirationen untersucht.

Einfluss des sozialen Hintergrunds Die Befunde zeigten, dass die tatsächlichen Korrekturen teilweise durch den sozialen Hintergrund beeinflusst wurden. Dementsprechend hat der Sprachgebrauch in der Familie einen substantiellen Einfluss auf das vorzeitige Verlassen des Weges zur Hochschulreife. Dafür sind verschiedene Erklärungen plausibel. Es ist möglich, dass Familien, die eine andere Sprache als Deutsch sprechen, ihre Fremdsprachenkenntnisse nutzen, die auf dem Weg zur Hochschulreife benötigt werden. Möglich ist aber auch, dass Schüler/innen aus fremdsprachigen Familien den allgemeinbildenden Weg sehr hoch einschätzen und den Wert der Berufsbildung unterschätzen. Darüber hinaus sei darauf hingewiesen, dass Aspirationen selbst auch durch die soziale Herkunft beeinflusst werden (vgl. Stubbe et al. 2012).

Weitere Einflussfaktoren In der vorliegenden Studie spielte die Leistung aus der Grundschule (Vergleichsprüfung) keine bzw. nur eine eingeschränkte Rolle für die Vorhersage der Korrekturen. Neuenschwander und Malti (2009) zeigten zwar für 
die Übergangsentscheidung nach der Sekundarstufe I (Gymnasium vs. Berufsausbildung), dass Noten und Leistungen aus der Grundschule eine prädiktive Kraft haben kontrollierten jedoch andere Variablen. Die Leistung aus der neunten Klasse war jedoch ein Prädiktor für die Aufwärtskorrektur. Anscheinend schätzen Schüler/-innen ihr Leistungsvermögen anhand der aktuellen Noten (der 9. Klasse) ein und treffen auf dieser Basis ihre Entscheidung. Des Weiteren könnten die Befunde auch dafür sprechen, dass einige Schüler/-innen ihre Leistungen während der Sekundarstufe I steigern konnten (,,late bloomers“). Es wurde auch aufgezeigt, dass die Noten in der neunten Klasse keine Rolle für die tatsächliche Abwärtskorrektur spielten. Jugendliche könnten möglicherweise die berufsbildende Anschlusslösung als gleichwertige Alternative zum Weg zur Hochschulreife sehen (vgl. Trautwein et al. 2008).

\subsection{Praktische und theoretische Implikationen}

Im Bildungssystem in Deutschfreiburg stellen „Korrekturen von zugewiesenen Bildungswegen“ (der Bildungsweg nach der Grundschule wurde nicht „frei“ gewählt) das Resultat langgehegter Aspirationen dar. In Bildungssystemen mit freiem Übergang nach der Grundschule könnten die „Korrekturen von eingeschlagenen Bildungswegen“"einerseits (a) auf eine Veränderung der Aspirationen hindeuten. Anderseits könnten Eltern den Übergang nach der Grundschule als Wahl einer zu diesem Zeitpunkt richtigen Entwicklungsumwelt verstanden und somit (b) ihre Aspirationen beibehalten haben. Aufgrund dieser unterschiedlichen Konzeptualisierung von „Korrekturen“ empfehlen wir gänzlich auf den Begriff der „Korrektur“ zu verzichten und lieber von Mobilität im Bildungssystem zu sprechen - wie es bereits in einigen Studien gemacht wird (z. B. Henz 1997; Buchholz und Schier 2015).

Die Ergebnisse der vorliegenden Studie sind - neben der aufgezeigten problematischen Verwendung des Korrektur-Begriffs - aus bildungspolitischer Sicht von Bedeutung. Die vertikale Öffnung hat dazu geführt, dass Bildungswege nicht mehr in ,Sackgassen“ enden, was eine wichtige Errungenschaft ist. Aufgrund der Vielzahl an nicht-linearen Bildungsverläufen kann das Bildungssystem in Deutschfreiburg als offen eingestuft werden. Bisher wurde die vertikale Öffnung aber auch mit Blick auf die Gefahr sinkender Leistungsstandards diskutiert (vgl. Baumert et al. 2003a). In Deutschfreiburg sollen teilweise Notenstandards dieser Gefahr entgegenwirken, allerdings haben grundsätzlich alle Schüler/-innen Möglichkeiten in die allgemeinbildende Anschlusslösung überzugehen (z. B. Zugang zur Fachmaturität). Die vorliegenden Befunde zeigen, dass der Notenschnitt der neunten Klasse beim Nachholen der Hochschulreife von Bedeutung ist. Dennoch sprechen die Ergebnisse - mit Bezug zu den Abwärtskorrekturen - für einen hoch attraktiven beruflichen Ausbildungssektor, der sich an leistungsstarke Absolventen der Sekundarstufe I wendet (vgl. Trautwein et al. 2008). Des Weiteren konnte die vorliegende Studie neue Erkenntnisse dazu liefern, dass es stark von den institutionellen Regelungen abhängt welche Determinanten auf Bildungsverläufe wirken (können). Im betrachteten Bildungswesen war der Einfluss der sozialen Herkunft gering. Darüber hinaus wurde aber gezeigt, dass Schüler/-innen ihre Aspirationen verwirklichen - die institutionellen Regelungen legen folglich den Zeitpunkt fest wann die Aspirationen umgesetzt werden können. 
Mit Blick auf zukünftige Forschung wären mehr Untersuchungen wünschenswert, die die institutionellen Rahmenbedingungen inkludieren. In diesem Zusammenhang wäre beispielsweise interessant herauszufinden, welche Rolle Aspirationen von verschiedenen Akteuren für Bildungsverläufe in Systemen mit weniger strikt geregelten ersten Übergangsverfahren spielen. Des Weiteren ist nach wie vor Forschung im Bereich der Aspirationen notwendig. Fraglich ist beispielsweise, ob realistische Aspirationen ähnlichen Einfluss haben, wie die hier verwendeten idealistischen Aspirationen (z.B. Kurz und Paulus 2008). Wie Becker (2010) anmerkte, könnte diese Aspirationsart vermehrt durch Kosten-Nutzen-Kalkulationen geprägt sein und damit eher zeitvariabel sein. Dementsprechend wären Forschungsarbeiten, die die zeitliche Stabilität von idealistischen und realistischen Aspirationen fokussieren, begrüßenswert. Schließlich sollten auch mögliche gegenseitige Einflüsse der Elternund Schüleraspirationen über mehrere Jahre hinweg näher erforscht werden.

\subsection{Grenzen der vorliegenden Studie}

Neben einer Vielzahl an Stärken dieser Studie, wie beispielsweise der Berücksichtigung zweier Operationalisierungen der Entscheidungen am Ende der Sekundarstufe I, des längsschnittlichen Designs (z.B. Vollerhebung zweier Jahrgänge in Deutschfreiburg) und Prädiktoren aus unterschiedlichen Disziplinen, müssen einige Schwächen aufgeführt werden: Wir analysierten die Bildungsentscheidungen nach der Sekundarstufe I - ob alle, die eine Aufwärtskorrektur vornahmen, anschließend auch erfolgreich die Hochschulzugangsberechtigung erworben haben, kann nicht festgestellt werden (vgl. Hillmert und Jacob 2010). Darüber hinaus besteht auch die Möglichkeit, dass Schüler/-innen zu einem späteren Zeitpunkt Korrekturen vornahmen - wir berücksichtigten nur die direkte Entscheidungen am Ende der Sekundarstufe I. An dieser Stelle möchten wir auch noch einmal darauf hinweisen, dass für die ,tatsächliche“ Entscheidung nach der Sekundarstufe I nur Angaben von der Kohorte 2010 vorlagen. Mit Blick auf die Aspirationen ist zu berücksichtigen, dass diese zwischen Eltern und Schüler/-innen geringfügig unterschiedlich erfasst wurden. Des Weiteren berücksichtigen wir nur die Aspirationen, die zum ersten Messzeitpunkt erhoben worden sind. Es sind somit keine Aussagen möglich, ob sich die Aspirationen der Akteure geändert haben. Abschließend können die Befunde der vorliegenden Studie nicht generalisiert werden und beziehen sich auf den deutschsprachigen Teil des Kantons Freiburg.

\section{Fazit}

Die vorliegende Studie zeigt, dass in Deutschfreiburg die Bildungsentscheidungen am Ende der Sekundarstufe I zwar „Korrekturen von zugewiesenen Bildungswegen“, gleichzeitig aber auch das Resultat langgehegter Aspirationen von Kindern und Eltern sind. Dementsprechend scheinen viele Schüler/-innen und Eltern den Bildungsweg seit der Grundschule festgelegt zu haben und sich nicht durch eine abweichende Zuweisung nach der Grundschule von diesem Plan abbringen zu las- 
sen. Somit hat die Zuweisung nach der Grundschule selbst scheinbar nur begrenzte Bedeutung für den weiteren individuellen Bildungsverlauf.

Danksagung Wir danken der Direktion für Erziehung, Kultur und Sport des Kantons Freiburg für ihre Unterstützung bei der Datenerhebung. Ebenso bedanken wir uns herzlich für wertvolle Kommentare und Hinweise bei der Schriftleitung und bei den zwei anonymen Gutachtern der Zeitschrift für Erziehungswissenschaft.

Open Access Dieser Artikel wird unter der Creative Commons Namensnennung 4.0 International Lizenz (http://creativecommons.org/licenses/by/4.0/deed.de) veröffentlicht, welche die Nutzung, Vervielfältigung, Bearbeitung, Verbreitung und Wiedergabe in jeglichem Medium und Format erlaubt, sofern Sie den/die ursprünglichen Autor(en) und die Quelle ordnungsgemäß nennen, einen Link zur Creative Commons Lizenz beifügen und angeben, ob Änderungen vorgenommen wurden.

Förderung Nele Usslepp ist Doktorandin des LEAD Graduate School \& Research Networks [GSC1028], welche durch die Exzellenzinitiative des Bundes und der Länder zur Förderung von Wissenschaft und Forschung an deutschen Hochschulen finanziert wird. Arbeiten am vorliegenden Beitrag wurden finanziert durch das Netzwerk Bildungsforschung der Baden-Württemberg Stiftung.

\section{Literatur}

Agresti, A. (2002). Categorical data analysis (2. Aufl.). New York: Wiley.

Baeriswyl, F., Wandeler, C., Trautwein, U., \& Oswald, K. (2006). Leistungstest, Offenheit von Bildungsgängen und obligatorische Beratung der Eltern. Zeitschrift für Erziehungswissenschaft, 9(3), 373-392. https://doi.org/10.1007/s11618-006-0056-6.

Baumert, J., Becker, M., Neumann, M., \& Nikolova, R. (2009). Frühübergang in ein grundständiges Gymnasium: Übergang in ein privilegiertes Entwicklungsmilieu? Ein Vergleich von Regressionsanalyse und Propensity Score Matching. Zeitschrift für Erziehungswissenschaft, 12(1), 189-215. https://doi. org/10.1007/s11618-009-0072-4.

Baumert, J., Cortina, K.S., \& Leschinsky, A. (2003a). Grundlegende Entwicklungen und Strukturprobleme im allgemein bildenden Schulwesen. In K. S. Cortina, J. Baumert, A. Leschinsky, K. U. Mayer \& L. Trommer (Hrsg.), Das Bildungswesen in der Bundesrepublik Deutschland: Strukturen und Entwicklungen im Überblick (S. 52-147). Reinbek: Rowohlt.

Baumert, J., Trautwein, U., \& Artelt, C. (2003b). Schulumwelten - institutionelle Bedingungen des Lehrens und Lernens. In Deutsches PISA-Konsortium (Hrsg.), PISA 2000: Ein differenzierter Blick auf die Länder der Bundesrepublik Deutschland (S. 261-331). Opladen: Leske + Budrich.

Becker, B. (2010). Bildungsaspiration von Migranten. Determinanten und Umsetzung in Bildungsergebnisse. Mannheim: Mannheimer Zentrum für Europäische Sozialforschung (MZES).

Becker, R. (2000). Bildungsexpansion und Bildungsbeteiligung. Zeitschrift für Erziehungswissenschaft, 3(3), 447-479. https://doi.org/10.1007/s11618-000-0043-2.

Becker, R., \& Hadjar, A. (2017). Meritokratie - Zur gesellschaftlichen Legitimation ungleicher Bildungs-, Erwerbs-und Einkommenschancen in modernen Gesellschaften. In R. Becker (Hrsg.), Lehrbuch der Bildungssoziologie (S. 33-62). Wiesbaden: Springer.

Becker, R., \& Zangger, C. (2013). Die Bildungsexpansion in der Schweiz und ihre Folgen. Eine empirische Analyse des Wandels der Bildungsbeteiligung und Bildungsungleichheiten mit den Daten der Schweizer Volkszählungen 1970, 1980,1990 und 2000. Kölner Zeitschrift für Soziologie und Sozialpsychologie, 65, 423-449. https://doi.org/10.1007/s11577-013-0209-6.

Best, H., \& Wolf, C. (2012). Modellvergleich und Ergebnisinterpretation in Logit-und Probit-Regressionen. KZfSS Kölner Zeitschrift für Soziologie und Sozialpsychologie, 64(2), 377-395. https://doi.org/ 10.1007/s11577-012-0167-4.

BMBF (2010). Der Übergang von der Grundschule in die weiterführende Schule. Leistungsgerechtigkeit und regionale, soziale und ethnische-kulturelle Disparitäten. https://www.bmbf.de/pub/ Bildungsforschung_Band_34.pdf. Zugegriffen: 21. März 2018.

Boudon, R. (1974). Education, opportunity, and social inequality: changing prospects in Western society. New York: Wiley.

Breen, R., \& Goldthorpe, J.H. (1997). Explaining educational differentials: towards a formal rational action theory. Rationality and Society, 9, 275-305. https://doi.org/10.1177/104346397009003002. 
Buchholz, S., \& Schier, A. (2015). New game, new chance? Social inequalities and upgrading secondary school qualifications in West Germany. European Sociological Review, 31(5), 603-615. https://doi. org/10.1093/esr/jcv062.

Buchholz, S., Skopek, J., Zielonka, M., Ditton, H., Wohlkinger, F., \& Schier, A. (2016). Secondary school differentiation and inequality of educational opportunity in Germany. In H.-P. Blossfeld, S. Buchholz, J. Skopek \& M. Triventi (Hrsg.), Models of secondary education and social inequality-an international comparison (S. 79-92). Cheltenham: Edward Elgar Publishing.

Bundesverfassung (1999). Art. 61, Abs. 1: Bundesverfassung der Schweizerischen Eidgenossenschaft. https://www.admin.ch/opc/de/classified-compilation/19995395/index.html\#a8 Stand: 2018.

Deutscher Bildungsrat (1970). Strukturplan für das Bildungswesen. Stuttgart: Klett.

Deutsches PISA-Konsortium (2001). PISA 2000. Basiskompetenzen von Schülerinnen und Schülern im internationalen Vergleich. Opladen: Leske + Budrich.

Ditton, H. (2013). Bildungsverläufe in der Sekundarstufe. Ergebnisse einer Längsschnittstudie zu Wechseln der Schulform und des Bildungsgangs. Zeitschrift für Pädagogik, 59(6), 887-911.

Ditton, H., \& Krüsken, J. (2010). Bildungslaufbahnen im differenzierten Schulsystem - Entwicklungsverläufe von Laufbahnempfehlungen und Bildungsaspirationen in der Grundschulzeit. In J. Baumert, K. Maaz \& U. Trautwein (Hrsg.), Bildungsentscheidungen (Zeitschrift für Erziehungswissenschaft: Sonderheft 12, S. 74-102). Wiesbaden: VS.

Ditton, H., Krüsken, J., \& Schauenberg, M. (2005). Bildungsungleichheit - der Beitrag von Familie und Schule. Zeitschrift für Erziehungswissenschaft, 8(2), 285-304. https://doi.org/10.1007/s11618-0050138-x.

Dumont, H., Maaz, K., Neumann, M., \& Becker, M. (2014). Soziale Ungleichheiten beim Übergang von der Grundschule in die Sekundarstufe I: Theorie, Forschungsstand, Interventions-und Fördermöglichkeiten. Zeitschrift für Erziehungswissenschaft, 17(2), 141-165.

EDK (1970). Konkordat über die Schulkoordination. https://edudoc.ch/record/1987/files/1-1d.pdf

EDK (2015a). Klassifikation der schweizerischen Bildungsstatistik (ISCED 2011). https://www.bfs.admin. ch/bfs/de/home/statistiken/bildung-wissenschaft/bildungssystem.assetdetail.248799.html. Zugegriffen: 21. März 2018.

EDK (2015b). Das Bildungswesen in der Schweiz (vereinfacht). https://www.bfs.admin.ch/bfs/de/home/ statistiken/bildung-wissenschaft/bildungssystem.html. Zugegriffen: 21. März 2018.

Enders, C. K. (2001). The impact of nonnormality on full information maximum-likelihood estimation for structural equation models with missing data. Psychological Methods, 6(4), 352-370. https://doi.org/ 10.1037//1082-989X.6.4.352-370.

Erikson, R., \& Jonsson, J. O. (1996). Can education be equalized? The Swedish case in comparative perspective. Boulder: Westview Press.

Gambetta, D. (1996). Were they pushed or did they jump? Individual decision mechanisms in education. Boulder: Westview Press.

Ganzeboom, H.B., \& Treiman, D. J. (1996). Internationally comparable measures of occupational status for the 1988 International Standard Classification of Occupations. Social science research, 25(3), 201-239. https://doi.org/10.1006/ssre.1996.0010.

Haller, A. O. (1968). On the concept of aspiration. Rural Sociology, 33(4), 484-487.

Haller, A. O., \& Portes, A. (1973). Status attainment process. Sociology of Education, 46(1), 51-91.

Harazd, B., \& Ophuysen, S. (2008). Was bedingt die Wahl eines nicht empfohlenen höheren Bildungsgangs? Zeitschrift für Erziehungswissenschaft, 11(4), 626-647. https://doi.org/10.1007/s11618-0080037-z.

Hauser, R. M. (2005). Survey response in the long run: The Wisconsin Longitudinal Study. Field Methods, 17(1), 3-29. https://doi.org/10.1177/1525822X04272452.

Henz, U. (1997). Der Beitrag von Schulformwechseln zur Offenheit des allgemeinbildenden Schulsystems. Zeitschrift für Soziologie, 26(1), 53-69. https://doi.org/10.1515/zfsoz-1997-0104.

Henz, U., \& Maas, I. (1995). Chancengleichheit durch die Bildungsexpansion. In H. Sahner, S. Schwendtner \& Deutsche Gesellschaft für Soziologie (DGS) (Hrsg.), 27. Kongreß der Deutschen Gesellschaft für Soziologie - Gesellschaften im Umbruch: Sektionen und Arbeitsgruppen (S. 389-393). Opladen: Westdeutscher Verlag.

Hillmert, S., \& Jacob, M. (2010). Selections and social selectivity on the academic track: A lifecourse analysis of educational attainment in Germany. Research in Social Stratification and Mobility, 28, 59-76. https://doi.org/10.1016/j.rssm.2009.12.006.

Hofstetter, D. (2017). Die schulische Selektion als soziale Praxis. Aushandlungen von Bildungsentscheidungen beim Übergang von der Primarschule in die Sekundarstufe I. Weinheim: Beltz Juventa. 
Kleine, L., Paulus, W., \& Blossfeld, H.-P. (2009). Die Formation elterlicher Bildungsentscheidungen beim Übergang von der Grundschule in die Sekundarstufe I. In J. Baumert, K. Maaz \& U. Trautwein (Hrsg.), Bildungsentscheidungen (Zeitschrift für Erziehungswissenschaft: Sonderheft 12, S. 103-125). Wiesbaden: VS.

Kuha, J., \& Mills, C. (2018). On group comparisons with logistic regression models. Sociological Methods \& Research, 1-28. https://doi.org/10.1177/0049124117747306.

Kurz, K., \& Paulus, W. (2008). Übergänge im Grundschulalter: Die Formation elterlicher Bildungsaspirationen. In K.S. Rehberg \& Deutsche Gesellschaft für Soziologie (DGS) (Hrsg.), Die Natur der Gesellschaft: Verhandlungen des 33. Kongresses der Deutschen Gesellschaft für Soziologie in Kassel 2006. Frankfurt a. M.: Campus.

Maaz, K., \& Nagy, G. (2009). Der Übergang von der Grundschule in die weiterführenden Schulen des Sekundarschulsystems: Definition, Spezifikation und Quantifizierung primärer und sekundärer Herkunftseffekte. In J. Baumert, K. Maaz \& U. Trautwein (Hrsg.), Bildungsentscheidungen (Zeitschrift für Erziehungswissenschaft: Sonderheft 12, S. 153-182). Wiesbaden: VS.

Maaz, K., Baeriswyl, F., \& Trautwein, U. (2011). Herkunft zensiert? Leistungsdiagnostik und soziale Ungleichheiten in der Schule; eine Studie im Auftrag der Vodafone Stiftung Deutschland. Düsseldorf: Vodafone Stiftung Deutschland.

McNeish, D., Stapleton, L. M., \& Silverman, R.D. (2017). On the unnecessary ubiquity of hierarchical linear modeling. Psychological Methods, 22(1), 114-140. https://doi.org/10.1037/met0000078.

Mood, C. (2010). Logistic regression: Why we cannot do what we think we can do, and what we can do about it. European sociological review, 26(1), 67-82. https://doi.org/10.1093/esr/jcp006.

Muthén, L. K., \& Muthén, B. O. (2012). Mplus user's guide (7. Aufl.). Los Angeles: Muthén \& Muthén.

Neuenschwander, M.P., \& Malti, T. (2009). Selektionsprozesse beim Übergang in die Sekundarstufe I und II. Zeitschrift für Erziehungswissenschaft, 12(2), 216-232. https://doi.org/10.1007/s11618-0090074-2.

Oesch, D. (2017). Potenzielle und realisierte Durchlässigkeit in gegliederten Bildungssystemen - Eine lokalstrukturelle Übertrittsanalyse in zwei Schulsystemen. Wiesbaden: VS.

Paulus, W., \& Blossfeld, H.-P. (2007). Schichtspezifische Präferenzen oder sozioökonomisches Entscheidungskalkül? Zur Rolle elterlicher Bildungsaspirationen im Entscheidungsprozess beim Übergang von der Grundschule in die Sekundarstufe. Zeitschrift für Pädagogik, 53(4), 491-508.

Sacchi, S., Hupka-Brunner, S., Stalder, B.E., \& Gangl, M. (2011). Die Bedeutung von sozialer Herkunft und Migrationshintergrund für den Übertritt in anerkannte nachobligatorische Ausbildungen in der Schweiz. In M. M. Bergman, S. Hupka-Brunner, A. Keller, T. Meyer \& B. E. Stalder (Hrsg.), Transitionen im Jugendalter. Ergebnisse der Schweizer Längsschnittstudie TREE (S. 120-156). Zürich: Seismo.

Schneider, T. (2008). Social inequality in educational participation in the German school system in a longitudinal perspective: Pathways into and out of the most prestigious school track. European Sociological Review, 24, 511-526. https://doi.org/10.1093/esr/jcn017.

Sewell, W.H., Haller, A. O., \& Ohlendorf, G.W. (1970). The educational and early occupational status attainment process: Replication and revision. American Sociological Review, 35(6), 1014-1027.

Sewell, W. H., Hauser, R. M., Springer, K. W., \& Hauser, T. S. (2003). As we age: a review of theWisconsin Longitudinal Study, 1957-2001. Research in Social Stratification and Mobility, 20, 3-111.

Stamm, M. (2005). Bildungsaspiration, Begabung und Schullaufbahn: Eltern als Erfolgspromotoren? Schweizer Zeitschrift für Bildungswissenschaften, 27(2), 277.

Stubbe, T.C., Bos, W., \& Euen, B. (2012). Der Übergang von der Primar- in die Sekundarstufe. In W. Bos, I. Tarelli, A. Bremerich-Vos \& K. Schwippert (Hrsg.), IGLU 2011. Lesekompetenzen von Grundschulkindern in Deutschland im internationalen Vergleich (S. 209-226). Münster: Waxmann.

Trautwein, U., \& Baeriswyl, F. (2007). Wenn leistungsstarke Klassenkameraden ein Nachteil sind: Referenzgruppeneffekte bei Übertrittsentscheidungen. Zeitschrift für Pädagogische Psychologie, 21(2), 119-133. https://doi.org/10.1024/1010-0652.21.2.119.

Trautwein, U., Baeriswyl, F., Lüdtke, O., \& Wandeler, C. (2008). Die Öffnung des Schulsystems: Fakt oder Fiktion? Zeitschrift für Erziehungswissenschaft, 11(4), 648-665. https://doi.org/10.1007/s11618008-0043-1.

UNESCO (2012). International Standard Classification of Education: ISCED 2011. Montreal: UNESCO Institute for Statistics (UIS).

Williams, R. (2015). Logistic regression, part II: the logistic regression models (LRM) - interpreting parameters. https://www3.nd.edu/ rwilliam/. Zugegriffen: 26. Sept. 2018.

Winkler, O. (2016). Aufstiege und Abstiege im Bildungsverlauf. Eine empirische Untersuchung zur Öffnung von Bildungswegen. Wiesbaden: VS. 
Wohlkinger, F., \& Ditton, H. (2012). Entscheiden die Schüler mit? Der Einfluss von Eltern, Lehrern und Kindern auf den Übergang nach der Grundschule. In R. Becker \& H. Solga (Hrsg.), Soziologische Bildungsforschung (Kölner Zeitschrift für Soziologie und Sozialpsychologie: Sonderheft 5, S. 44-63). Wiesbaden: Springer.

Zimmermann, T. (2018). Die Bedeutung signifikanter Anderer für eine Erklärung sozial differenzierter Bildungsaspirationen. Zeitschrift für Erziehungswissenschaft, 21(2), 339-360. https://doi.org/10.1007/ s11618-017-0781-z. 